\title{
Particle image velocimetry measurements of the interaction of synthetic jets with a zero-pressure gradient laminar boundary layer
}

\author{
Mark Jabbal ${ }^{\mathrm{a})}$ and Shan Zhong \\ School of Mechanical, Aerospace and Civil Engineering, University of Manchester, \\ Manchester M60 1QD, United Kingdom
}

(Received 9 August 2009; accepted 20 April 2010; published online 21 June 2010)

\begin{abstract}
An experimental investigation of the interaction between a synthetic jet actuator and a zero-pressure gradient laminar boundary layer is reported. The aim of this study is to quantify the impact of synthetic jet vortical structures; namely, hairpin vortices, stretched vortex rings and tilted vortex rings on a boundary layer, and to assess their relative potential for flow separation control. Streamwise particle image velocimetry was employed in a water flume (free stream boundary layer thickness Reynolds number of 500 and boundary layer thickness-to-jet orifice diameter ratio of 4) to obtain phase- and time-averaged boundary layer profile information of the impact of synthetic jets near the wall. The potential for flow control was assessed by analyzing near wall fluid mixing, realized by the measure of increase in wall shear stress produced by a passing vortex. Hairpin vortices (produced at a jet-to-free stream velocity ratio, $V R=0.32$ and dimensionless stroke length, $L=1.6)$ and stretched vortex rings $(V R=0.27 ; L=2.7)$ exhibit characteristics akin to a streamwise vortex pair with a common upwash. Conversely, tilted vortex rings $(V R=0.54 ; L=2.7)$ induce a streamwise vortex pair in the near wall region with a common downwash. Wall shear stress measurements show that synthetic jets composed of stretched vortex rings offer the best combination of near wall fluid mixing, persistency, and low rms fluctuations for potential applications of flow separation control. (C) 2010 American Institute of Physics.
\end{abstract}

[doi:10.1063/1.3432133]

\section{INTRODUCTION}

Forecasts in the growth of air traffic, carbon emissions, and fuel prices have placed high demands on the aviation industry to improve aerodynamic efficiency and minimize operating costs. In Europe, the EU has coordinated a response to this challenge primarily through the Advisory Council for Aeronautics Research in Europe (ACARE). The ACARE 2020 Vision calls for a 50\% reduction in $\mathrm{CO}_{2}$ and an $80 \%$ reduction in $\mathrm{NO}_{\mathrm{X}}$ emissions per passenger kilometer. ${ }^{1}$ One of the key points in the ACARE strategy is the focus on developing greener aircraft by bringing a number of cutting edge technologies through to full demonstration. The development of active flow control (AFC) technologies is one such major example.

Synthetic jet actuators (SJAs) are a promising form of AFC technology, with a proven capability of demonstrating flow separation control at laboratory scale. ${ }^{2-4}$ Wood et al. demonstrated the flow separation control effectiveness of an array of round SJAs on a two-dimensional (2D) circular cylinder in a turbulent boundary layer. ${ }^{5}$ Surface oil visualization showed that with the actuators active, the separation line was pushed downstream noticeably.

In this type of actuator, electrical power is converted into fluidic power by the mechanical excitation of an oscillating diaphragm on one or both sides of a cavity (Fig. 1). A jet is synthesized from the surrounding fluid due to suction and expulsion at the orifice exit. These zero-net-mass-flux actua-

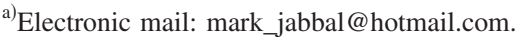

tors negate the need for bleed air supply and piping associated with pneumatic devices. SJAs, therefore, open up possibilities for the efficient, low-energy input control of separated flows on aircraft, with the potential to offer improvements in aerodynamic performance. Ultimately, this could lead to reduced fuel consumption and reductions in the release of environmentally deleterious emissions.

Despite the promise of SJAs, little is still known about the detailed flow physical processes of interaction between a SJA and a boundary layer. For practical flow control purposes, the typical vortical structures formed by the interaction of synthetic jets with a boundary layer, their impact near the wall and their relative effectiveness must be fully understood. To date, there have been many experimental and numerical investigations aimed at improving understanding of the interaction between a synthetic jet and a boundary layer. A summary of some of these studies ${ }^{6-14}$ is provided in Table I. Although the studies in Table I are not demonstrations of flow control per se, none offer a detailed insight into the type of vortical structures generated by the interaction or an assessment of their potential effectiveness for separation control.

Flow visualizations have been carried out to examine the type of vortical structures produced as a result of the interaction between synthetic jets and a flat plate laminar boundary layer. ${ }^{15}$ Three distinct behaviors were observed. At low jet-to-free stream velocity ratios $V R$ and jet Reynolds numbers $\mathrm{Re}_{L}$, the vortical structures produced by synthetic jets appear as hairpin vortices, which are attached to the wall. At intermediate $V R$ and $\mathrm{Re}_{L}$, the vortex sheet produced at the 


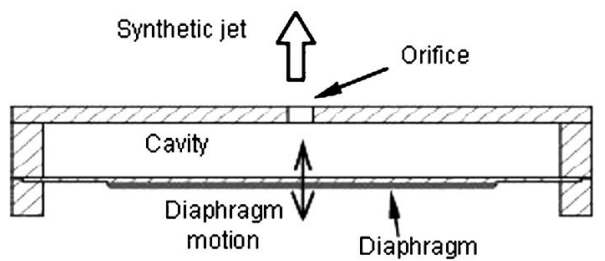

FIG. 1. A generic SJA.

orifice rolled up into vortex rings, which experienced considerable tilting and stretching as they enter the boundary layer. At high $V R$ and $\mathrm{Re}_{L}$, the vortex rings appeared to experience a certain amount of tilting but no obvious stretching and penetrate the edge of the boundary layer quickly.

Further work was carried out to investigate the impact of the vortical structures at the wall, where effective flow control is ultimately desired. ${ }^{16}$ The experiments were conducted with a temperature-sensitive liquid crystal (TLC) coating applied on the test surface. The TLC images showed two distinct patterns of thermal footprints depending on the nature of the flow structures that produce them. It was found that both hairpin vortices and stretched vortex rings each produced two streamwise streaks of high heat transfer which, by Reynolds analogy, corresponds to the transfer of high momentum fluid toward the wall outboard of the streamwise counter-rotating legs. In contrast, the tilted vortex rings pro- duced only a single streamwise streak of high heat transfer, which was hypothesized to be a result of an induced vortex adjacent to the wall. The different vortical structures of synthetic jets were mapped in nondimensional parameter space for the first time. ${ }^{16}$

In view of the similarity in their footprints to the oil flow pattern on the $2 \mathrm{D}$ circular cylinder model, ${ }^{5}$ both the hairpin vortices and stretched vortex rings appear capable of producing the well-defined streamwise vortex pairs that delayed flow separation on the cylinder. In spite of these qualitative findings, however, quantitative measurements are ultimately required to identify the relative effectiveness of the vortical structures for separation control.

In this paper, the results of a quantitative investigation of the interaction of a SJA with the near wall region of a boundary layer are presented. The first objective of this study is to quantify the impact of the typical vortical structures on the boundary layer velocity profiles. The second objective of this study is to analyze the near wall fluid mixing capability of each vortical structure and in doing so, identify effective vortical structures with potential for flow separation control. In order to achieve these objectives, high frame rate particle image velocimetry (PIV) was used.

As a continuation of the authors' qualitative study, ${ }^{16}$ a single SJA embedded in a flat plate under the influence of a zero-pressure gradient laminar boundary layer was chosen. Despite the flow over aircraft high-lift devices being turbu-

TABLE I. Effective parameters and observations in previous SJA-boundary layer studies. (ZPGBL=zero-pressure gradient boundary layer; APGBL $=$ adverse-pressure gradient boundary layer; $\operatorname{Re}_{\delta}=$ Reynolds number based on free stream velocity and boundary layer thickness; $V R=$ jet-to-free stream velocity ratio based on peak jet velocity; $C_{\mu}=$ momentum coefficient based on peak jet velocity, orifice diameter/width and plate length; $L=$ dimensionless stroke length; $\mathrm{St}=$ free stream Strouhal number; and $d=$ boundary layer thickness-to-orifice diameter/width ratio.)

\begin{tabular}{|c|c|c|c|c|c|c|c|c|c|}
\hline Study & Model & Orifice & $\operatorname{Re}_{\delta}$ & $V R$ & $C_{\mu}$ & $L$ & St & $d$ & $\begin{array}{l}\text { Observations/ } \\
\text { hypothesized vortices }\end{array}$ \\
\hline $\begin{array}{l}\text { Mittal et al. }{ }^{\text {a }} \\
2001\end{array}$ & $\begin{array}{c}\text { Laminar ZPGBL } \\
\text { (simulation-flat plate) }\end{array}$ & Slot & $\begin{array}{c}2600 \\
1200 \\
260\end{array}$ & $\begin{array}{l}0.3 \\
0.67 \\
3\end{array}$ & $\cdots$ & $\cdots$ & $\ldots$ & 2 & $\begin{array}{l}\text { Embedded asymmetric vortex; } \\
\text { asymmetric vortex with entrainment; } \\
\text { vortex pair penetrating into free stream }\end{array}$ \\
\hline $\begin{array}{l}\text { Smith }^{\mathrm{b}} \\
2002\end{array}$ & $\begin{array}{l}\text { Turbulent ZPGBL } \\
\text { (flat plate) }\end{array}$ & Slot & 18000 & 1.2 & 0.012 & 13 & 0.1 & 56 & $\begin{array}{c}\text { Consistent with the presence of embedded } \\
\text { longitudinal vortices }\end{array}$ \\
\hline $\begin{array}{l}\text { Cui et } a l^{\mathrm{c}} \\
2003\end{array}$ & $\begin{array}{c}\text { Turbulent ZPGBL } \\
\text { (simulation-flat plate) }\end{array}$ & Slot & $\begin{array}{c}7000 \\
21400\end{array}$ & $\begin{array}{l}3.9 \\
3.9\end{array}$ & $\begin{array}{l}0.058 \\
0.016\end{array}$ & $\begin{array}{l}17 \\
22\end{array}$ & $\begin{array}{l}0.07 \\
0.06\end{array}$ & $\begin{array}{l}6.6 \\
61\end{array}$ & $\begin{array}{l}\text { Discrete vortices scale with } \delta \text {; } \\
\text { discrete vortices scale with inner sublayer }\end{array}$ \\
\hline $\begin{array}{l}\text { Wu and Breuer } \\
2003\end{array}$ & $\begin{array}{l}\text { Turbulent ZPGBL } \\
\text { (flat plate) }\end{array}$ & Slot & $\cdots$ & 0.05 & $\cdots$ & $0.25-1$ & 0.02 & $\cdots$ & $\begin{array}{c}\text { No discrete vortices } \\
\text { (emphasis on turbulence control) }\end{array}$ \\
\hline $\begin{array}{l}\text { Shuster } \text { et }_{\text {al. }}{ }^{\mathrm{e}} \\
2005\end{array}$ & $\begin{array}{l}\text { Laminar ZPGBL } \\
\quad \text { (flat plate) }\end{array}$ & Hole & $\cdots$ & 1.06 & 0.023 & $\begin{array}{l}1 \\
2\end{array}$ & $\cdots$ & $\cdots$ & $\begin{array}{l}\text { Asymmetric vortex; } \\
\text { symmetric vortex ring }\end{array}$ \\
\hline $\begin{array}{l}\text { Schaeffler } \text { et } a l^{\mathrm{f}} \\
2006\end{array}$ & $\begin{array}{l}\text { Turbulent ZPGBL } \\
\text { (flat plate) }\end{array}$ & Hole & 50100 & $\begin{array}{l}0.56 \\
1.3\end{array}$ & $\cdots$ & $\begin{array}{l}\cdots \\
\cdots\end{array}$ & $\begin{array}{l}0.24 \\
0.49\end{array}$ & 3.3 & Generation of vortical structures \\
\hline $\begin{array}{l}\text { Dandois et al. }{ }^{\mathrm{g}} \\
2006\end{array}$ & $\begin{array}{c}\text { Turbulent ZPGBL } \\
\text { (simulation-flat plate) }\end{array}$ & Hole & 45500 & 1.45 & 0.175 & 17 & 0.03 & 3 & $\begin{array}{l}\text { Counter-rotating vortex pair with ring } \\
\text { vortices and horseshoe vortex }\end{array}$ \\
\hline $\begin{array}{l}\text { Hong }^{\mathrm{h}} \\
2006\end{array}$ & $\begin{array}{l}\text { Laminar APGBL } \\
\quad \text { (flat plate) }\end{array}$ & Hole & 500 & 0.75 & 0.0012 & 38 & 0.006 & 2 & $\begin{array}{c}\text { Nondescriptive vortices } \\
\text { (emphasis on promoting transition) }\end{array}$ \\
\hline $\begin{array}{l}\text { Wu and Leschziner }{ }^{\mathrm{i}} \\
2008\end{array}$ & $\begin{array}{c}\text { Turbulent ZPGBL } \\
\text { (simulation-flat plate) }\end{array}$ & Hole & 9000 & 2 & 0.057 & 9 & 0.07 & 4 & Streamwise vortex pair \\
\hline
\end{tabular}

${ }^{\mathrm{a}}$ Reference 6.

${ }^{\mathrm{b}}$ Reference 7.

${ }^{\mathrm{c}}$ Reference 8 .

${ }^{\mathrm{d}}$ Reference 9 .

${ }^{\mathrm{e}}$ Reference 10 .
${ }^{\mathrm{f}}$ Reference 11 .

${ }^{\mathrm{g}}$ Reference 12 .

${ }^{\mathrm{h}}$ Reference 13 .

${ }^{\mathrm{i}}$ Reference 14. 
lent, which is a candidate application for SJAs, the use of a zero-pressure gradient laminar boundary layer provides a logical initial step toward fully understanding the synthetic jet-boundary layer interaction. Furthermore, it is believed by the authors that the types of vortical structures generated as a result of the interaction with a laminar flow are essentially the same as those in a turbulent flow. ${ }^{17}$ The qualitative similarities between the synthetic jet footprints in a zero-pressure gradient laminar boundary layer ${ }^{16}$ and a separating turbulent boundary layer ${ }^{5}$ appear to support this hypothesis.

Such a fundamental study, of course, does not allow for a direct comparison of the relative effectiveness of the synthetic jet vortical structures in delaying separation. The question that therefore arises is how to compare the potential effectiveness of the vortical structures for separation control. In a study of vane vortex generators for the control of an adverse-pressure gradient boundary layer, ${ }^{18}$ an "optimal" coherent structure for separation control was defined as one that transfers momentum toward the wall, increasing skin friction, i.e., wall shear stress. It was similarly acknowledged $^{19}$ that regions of significant wall shear stress enhancement associated with the downwash generated by longitudinal vortices in a zero-pressure gradient turbulent boundary layer are important because they indicate the ability of the vortices to forestall boundary layer separation. On this basis, the criteria for assessing the relative effectiveness of the synthetic jet vortical structures was made from the level of wall shear stress enhancement and the persistence of this enhancement in the boundary layer.

\section{EXPERIMENTAL APPROACH}

PIV measurements were conducted in a $4 \mathrm{~m}$ water flume. The test section of the flume is $0.8 \mathrm{~m}$ in length with a cross section of $0.3 \times 0.3 \mathrm{~m}^{2}$. A uniform steady flow with a velocity up to $0.4 \mathrm{~m} / \mathrm{s}$ can be achieved in the test section. The SJA used in this investigation consisted of a cylindrical cavity bounded by rigid sidewalls with an orifice plate at one end and a diaphragm clamped to the other. The cavity has a diameter, $D_{c}=45 \mathrm{~mm}$ and height, $H=25 \mathrm{~mm}$. The orifice has a diameter, $D_{o}=5 \mathrm{~mm}$ and depth, $h=5 \mathrm{~mm}$. The arrangement of the SJA embedded in the boundary layer plate is the same as that described for the dye visualization tests. ${ }^{16}$

\section{A. PIV measurements}

To facilitate PIV measurements, the free stream and SJA internal flow were seeded with Dantec Dynamics hollow glass particles with a mean diameter of $10 \mu \mathrm{m}$ and density of $1100 \mathrm{~kg} / \mathrm{m}^{3}$. The seeding particles were chosen because of their similar density to water. Based on Stokes drag law, the settling velocity of the particles was estimated to be $5 \mu \mathrm{m} / \mathrm{s}$. In addition, the ratio of particle relaxation time to the oscillation period of the synthetic jet cycle is of the order of $10^{-5}$ for $f=2 \mathrm{~Hz}$ and smaller for $f=1 \mathrm{~Hz}$. These results, therefore, suggest that the particles will follow the motion of both the free stream and synthetic jet flow with high fidelity.

A $1 \mathrm{~mm}$ thick light sheet was generated by a $5 \mathrm{~W}$ argon ion laser to illuminate the flow field, in the first instance, along a streamwise plane that bisects the SJA orifice along

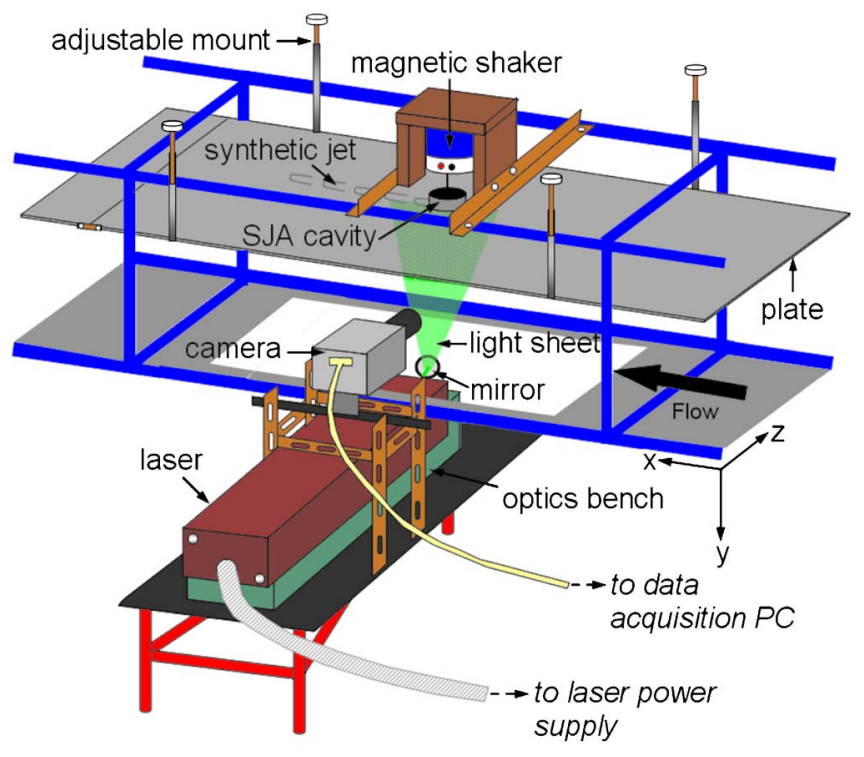

FIG. 2. (Color online) Schematic layout of experimental set up for PIV measurements in the tilting flume test section.

its centerline (Fig. 2). A $1024 \times 1024$ pixel Photron PCI CMOS camera with a Nikon $105 \mathrm{~mm}$ lens and Sigma $2 \times$ teleconverter were used to capture images in a 25 $\times 25 \mathrm{~mm}^{2}$ field of view, which was sufficient to cover the height of the undisturbed boundary layer. Corresponding vectors were resolved using a two-frame cross-correlation algorithm in which, a $32 \times 32$ pixel interrogation area with an overlap ratio of $50 \%$ was chosen, giving a spatial resolution of $0.39 \mathrm{~mm}$.

Centerline PIV measurements of synthetic jets were taken at two streamwise locations-in the near field $\left(-1 \leq \mathrm{x} / D_{o} \leq 4\right)$ and far field $\left(20 \leq \mathrm{x} / D_{o} \leq 25\right)$. For phaseaveraged measurements, the camera frame rate was selected according to the maximum jet velocity at the orifice. Measurements were taken at 250 and 500 equally spaced phase points across the oscillation cycle for a SJA frequency of $f=2$ and $1 \mathrm{~Hz}$, respectively, thereby giving temporal resolutions of $1.44^{\circ}$ and $0.72^{\circ}$. For a given phase, phase-averaged flow fields were obtained from a minimum of 60 instantaneous image pairs. Long time-averaged measurements of synthetic jets encompassing 6000 consecutively captured images were obtained in both near field and far field regions.

For off-center PIV measurements, the lightsheet was traversed in the spanwise direction (along the $\mathrm{z}$-axis in the coordinates shown in Fig. 2) at both streamwise locations. This was achieved by traversing the laser mirror along the optics bench. In the near field location, six streamwise planes spaced $1 \mathrm{~mm}$ apart (i.e., $0.2 D_{o}$ ) on each side of the centerline plane were recorded. In the far field, five streamwise planes spaced $2 \mathrm{~mm}$ apart (i.e., $0.4 D_{o}$ ) on each side of the centerline plane were recorded. A schematic of all the quantitative measurement planes is shown in Fig. 3.

The accuracy of the PIV measurements was assessed in accordance with continuity. ${ }^{20}$ Maximum uncertainties in the long-time averaged and phase-averaged velocities were $1.3 \%$ and $4.4 \%$, respectively. The uncertainty in vorticity was calculated by substituting the phase-averaged velocity uncer- 


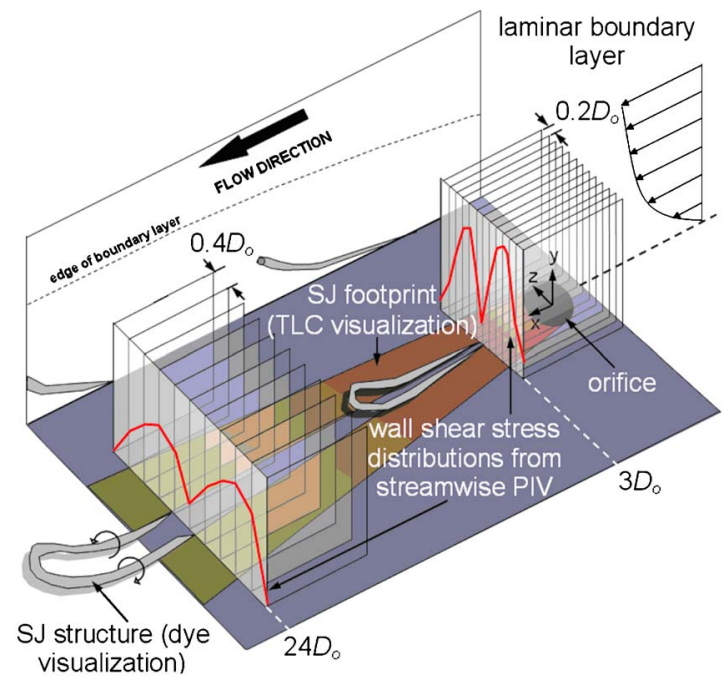

FIG. 3. (Color online) Schematic showing dye visualization, liquid crystal visualization and PIV measurement planes for a synthetic jet in a boundary layer.

tainties into the equation for vorticity at the center of a vortex. $^{20}$ Maximum uncertainty in vorticity relative to the peak value was calculated to be $5.5 \%$. Measurements of the undisturbed boundary layer thickness and wall shear stress were obtained from the long time-averaged velocity profiles. Relative to the Blasius solution, the maximum uncertainty in the boundary layer thickness was $2.2 \%$. For wall shear stress, measurement of the velocity gradient was made by taking a line of best fit through several points of the profile adjacent to the wall. Relative to the Blasius solution, the maximum uncertainty in wall shear stress was $6 \%$ for the near field measurements and $11 \%$ for the far field measurements.

\section{B. Test conditions}

The test section free stream velocity was fixed at $0.05 \mathrm{~m} / \mathrm{s}$ to allow for a comparison of the relative effectiveness of each vortex type in terms of wall shear stress enhancement. A good agreement of the undisturbed boundary layer velocity profile with the Blasius solution at $U_{\infty}=0.05 \mathrm{~m} / \mathrm{s}$ was confirmed in Fig. 4 . The boundary layer thickness-to-orifice diameter ratio, $d$ and skin friction coefficient, $c_{f}$ at the orifice location were 3.6 and $4.13 \times 10^{-3}$, respectively. Reynolds number based on the free stream velocity and boundary layer thickness $\operatorname{Re}_{\delta}$ was 500 .

Table II lists the cases investigated, which are representative of the three typical vortical structures produced by the interaction of a synthetic jet and a boundary layer. These structures were previously identified in nondimensional parameter space, ${ }^{16}$ namely, a hairpin vortex, stretched vortex ring and tilted vortex ring, where $f$ and $\Delta$ are the diaphragm oscillation frequency and peak-to-peak displacement, respectively. The jet-to-free stream velocity ratio, $V R$, is defined as

$$
V R=\frac{\bar{U}_{o}}{U_{\infty}},
$$

where $\bar{U}_{o}$ is the time-averaged blowing velocity over the entire cycle, which was used to compare synthetic jets with steady jets. ${ }^{21}$ In the present study

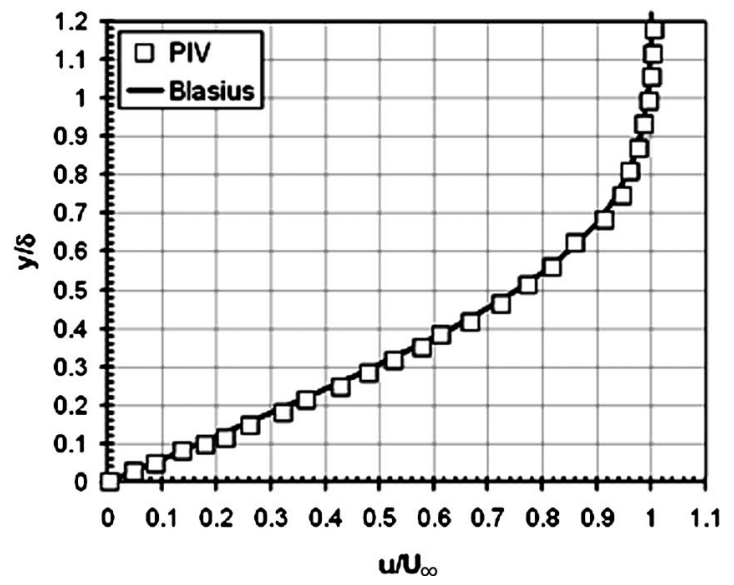

FIG. 4. Undisturbed boundary layer velocity profiles along a streamwise centerline location at $\mathrm{x} / D_{o}=-5$ for $U_{\infty}=0.05 \mathrm{~m} / \mathrm{s}$.

$$
\bar{U}_{o}=\frac{1}{T} \int_{0}^{T / 2} \tilde{u}_{o}(t) d t=\frac{1}{3} f \Delta\left(\frac{D_{c}}{D_{o}}\right)^{2},
$$

where $\tilde{u}_{o}(t)$ is the instantaneous space-averaged velocity at the orifice exit and $T$ is the time period of a complete oscillation cycle. For a sinusoidal diaphragm oscillation, the maximum space-averaged jet blowing velocity, $u_{\text {peak }}=\pi \bar{U}_{o}$. Assuming an incompressible flow, Eq. (2) equates the instantaneous volume flow rate displaced by the diaphragm with that expelled through the orifice. ${ }^{22}$ For completion, values of the jet momentum coefficient (based on $u_{\text {peak }}$, orifice diameter and flat plate length) for each case are also included in Table II.

The nondimensional stroke length, $L$, is defined as

$$
L=\frac{L_{o}}{D_{o}}=\frac{\bar{U}_{o}}{f D_{o}}
$$

where $L_{o}$ is the stroke length, which according to the slug model, ${ }^{23}$ represents the length of the jet fluid column expelled during blowing.

The Reynolds number based on the stroke length, $\operatorname{Re}_{L}$, is defined as

$$
\operatorname{Re}_{L}=\frac{\bar{U}_{o} L_{o}}{\nu} .
$$

Finally, Strouhal number based on local boundary layer thickness St is defined as

TABLE II. Case summary for PIV tests.

\begin{tabular}{lccccccc}
\hline \hline & $f$ & $\Delta$ & & & & & \\
Structure type & $(\mathrm{Hz})$ & $(\mathrm{mm})$ & $V R$ & $C_{\mu}$ & $L$ & $\mathrm{Re}_{L}$ & $\mathrm{St}$ \\
\hline $\begin{array}{l}\text { Hairpin } \\
\text { vortex }\end{array}$ & 2 & 0.3 & 0.32 & 0.0078 & 1.6 & 131 & 0.72 \\
$\begin{array}{l}\text { Stretched } \\
\text { vortex ring }\end{array}$ & 1 & 0.5 & 0.27 & 0.0055 & 2.7 & 182 & 0.36 \\
$\begin{array}{l}\text { Tilted } \\
\text { vortex ring }\end{array}$ & 2 & 0.5 & 0.54 & 0.022 & 2.7 & 364 & 0.72 \\
\hline \hline
\end{tabular}




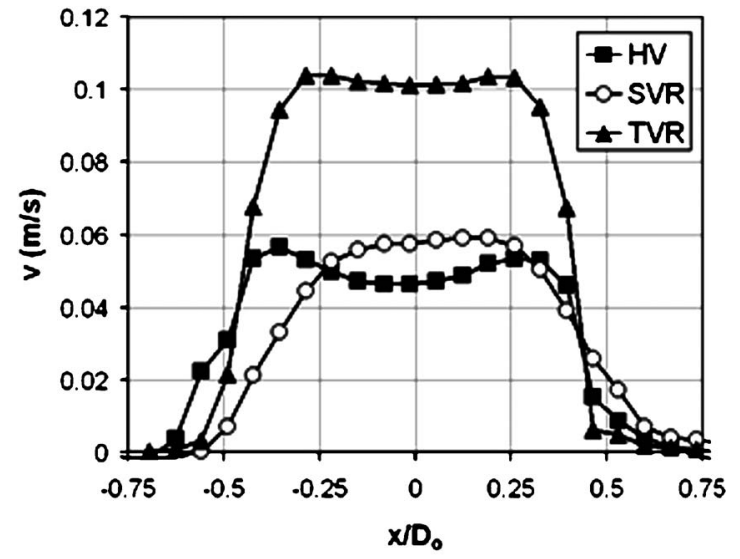

FIG. 5. Phase-averaged jet exit velocity profiles at maximum blowing $(\mathrm{HV}=$ hairpin vortex, $\mathrm{SVR}=$ stretched vortex ring, and $\mathrm{TVR}=$ tilted vortex ring).

$$
\mathrm{St}=\frac{f \delta}{U_{\infty}} .
$$

Assuming that the vortical structures travel at the free stream velocity, the inverse of St provides a measure of the streamwise spacing between consecutive structures as a fraction of the local boundary layer thickness.

A LabVIEW virtual instrument was used to generate a sinusoidal waveform for the SJA diaphragm at the specified oscillation frequency. The diaphragm displacement was measured using an eddy current displacement sensor. To ensure operation at the nondimensional parameters in Table II, measurements of jet velocity were extracted from phaseaveraged velocity profiles at the orifice. The jet profiles are shown in Fig. 5. The edge of the orifice corresponds to $\mathrm{x} / D_{o}=-0.5$ and 0.5 . For each case, $u_{\text {peak }}$ was obtained across the orifice exit. This velocity was compared with Eq. (2) and is shown in Table III. There is reasonable agreement between experiment and theory, with maximum uncertainty less than $10 \%$. The small discrepancy is likely to be a result of taking measurements within $0.12 D_{o}$ of the orifice exit, due to the difficulty in obtaining data exactly on the orifice exit plane, i.e., at $\mathrm{y} / D_{o}=0$.

\section{IMPACT OF SYNTHETIC JETS ON THE BOUNDARY LAYER}

\section{A. Qualitative summary}

Figure 6 shows visualization of the three types of synthetic jet vortical structures in Table II. Each case shows stereoscopic dye flow visualization and surface visualization of the vortex footprints using TLCs.

TABLE III. Comparison of the experimental and theoretical space-averaged jet velocities at the orifice exit.

\begin{tabular}{lccc}
\hline \hline Structure type & $\begin{array}{c}u_{\text {peak }} \\
(\mathrm{m} / \mathrm{s})(\mathrm{PIV})\end{array}$ & $\begin{array}{c}u_{\text {peak }} \\
(\mathrm{m} / \mathrm{s})[\mathrm{Eq} .(2)]\end{array}$ & $\begin{array}{c}\text { Difference } \\
(\%)\end{array}$ \\
\hline Hairpin vortex & 0.0464 & 0.0509 & 9.6 \\
Stretched vortex ring & 0.0442 & 0.0424 & 4.2 \\
Tilted vortex ring & 0.0828 & 0.0848 & 2.5 \\
\hline \hline
\end{tabular}

Two distinct types of thermal footprints are observed. Both hairpin vortices and stretched vortex rings each produce two streamwise streaks of high heat transfer (dark/red regions), which by Reynolds analogy, corresponds to high momentum fluid transfer toward the wall outboard of the streamwise counter-rotating legs. In contrast, the tilted vortex rings produced only a single streamwise streak of high heat transfer, which is hypothesized to be a result of an induced vortex adjacent to the wall. Note that the thermal footprints could not be used to compute wall shear stress since sufficient hue data could not be extracted from the TLC test surface. Consequently, a hue-temperature in situ calibration could not be made to determine heat transfer coefficient and hence wall shear stress distributions.

Figure 7 shows the trajectory of the vortical structures obtained from the positions of the vortices in dye flow. Both hairpin vortices and stretched vortex rings have quite similar trajectories due to similar jet-to-free stream velocity ratios, $V R$. The hairpin vortices remain fully embedded in the boundary layer, which gives rise to the high level of streamwise stretching seen in Fig. 6(a). On the other hand, the stretched vortex rings eventually penetrate the boundary layer at $\mathrm{x} / D_{o} \approx 9$, but still remain close to the edge of the boundary layer. The difference in trajectories arises from the lower actuation frequency used to generate the stretched vortex rings, which reduces the spacing and therefore interaction between consecutive structures. This results in increased penetration through the boundary layer. Due to the relatively high $V R$ used to generate tilted vortex rings, the vortices penetrate out of the boundary layer within a short distance downstream. Consequently, the tilted vortex rings undergo no appreciable stretching due to their short resident time in the boundary layer.

\section{B. Phase-averaged boundary layer profiles}

To gain an insight into the transient influence of hairpin vortices, stretched vortex rings and tilted vortex rings on the boundary layer, phase-averaged centerline velocity profiles were analyzed. Four phases for each case were arbitrarily chosen to cover the time period when the vortical structure passes across the $\mathrm{x} / D_{o}=3$ plane. For reference, the phases $t=0 \mathrm{~T}$ corresponds to the onset of jet blowing, $t=0.25 \mathrm{~T}$ maximum jet blowing, $t=0.50 \mathrm{~T}$ onset of jet suction, and $t=0.75 \mathrm{~T}$ maximum jet suction.

Note that for the following profiles (and subsequent long time-averaged profiles), the y-distance normal to the wall is nondimensionalized by the local undisturbed boundary layer height, $\delta$. The u-velocity component is in dimensional form since for certain cases, the edge of the boundary layer is displaced below the PIV field of view by the synthetic jet. Therefore, an exact measure of the free stream velocity at the edge of the boundary layer is not available to normalize velocity. For consistency, the u-velocity component is left in dimensional form for all cases. To aid interpretation, corresponding vorticity contours with an overlay of uniform velocity vectors are shown adjacent to each profile.

Figure 8 shows the phase-averaged boundary layer velocity profiles for a passing hairpin vortex. At $t=\mathrm{T} 1$, two 

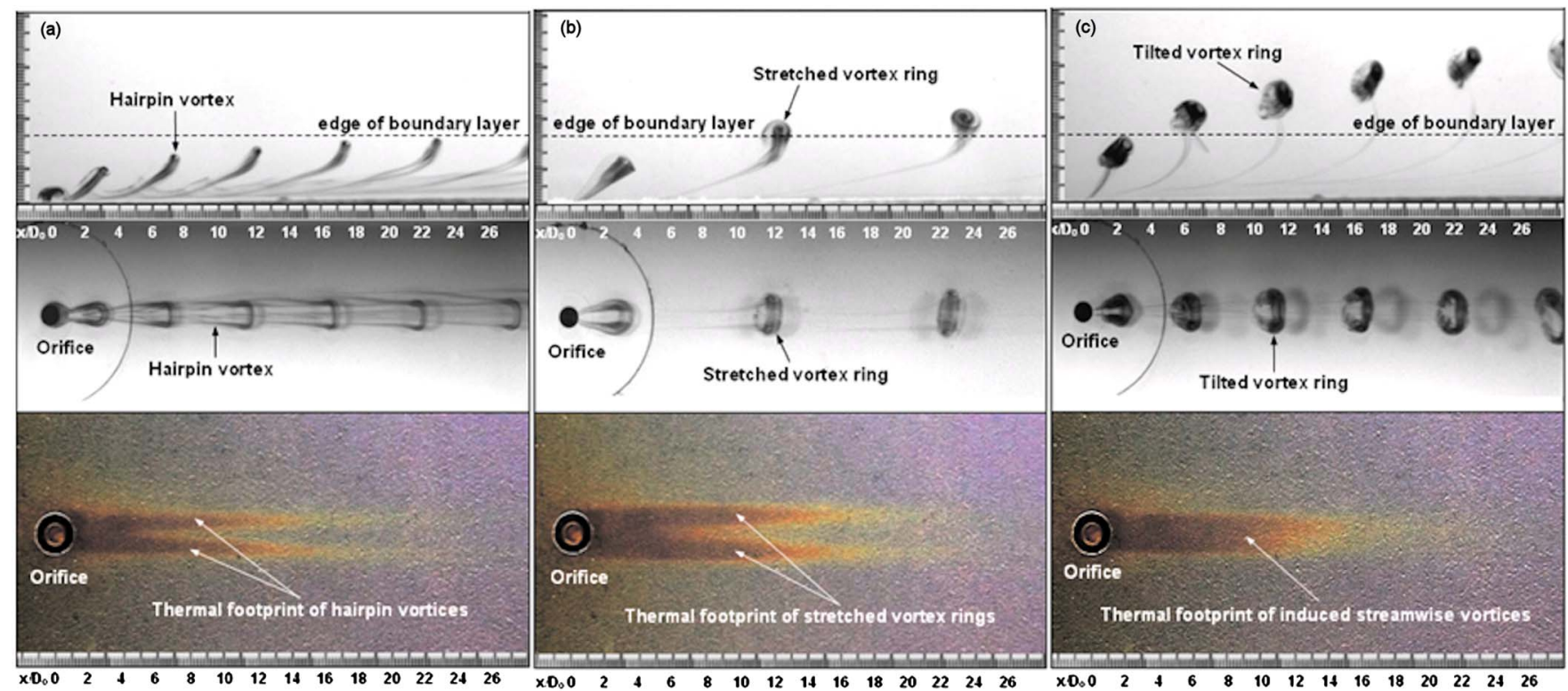

FIG. 6. (Color online) Flow visualization and thermal footprints of (a) hairpin vortices, (b) stretched vortex rings, and (c) tilted vortex rings.

vortical structures are observed in the field of view. A newly formed structure with a clockwise (negative) vorticity at the downstream side of the orifice and an anticlockwise (positive) vorticity at the upstream side of the orifice is seen in the vicinity of the orifice. This structure is in fact a young vortex ring. A second vortical structure formed in the previous cycle is approaching the $\mathrm{x}-\mathrm{y}$ plane at $\mathrm{x} / D_{o}=3$. The upstream branch of this vortex has noticeably weakened due to the countervorticity of the boundary layer to give the structure its characteristic asymmetric rollup. Interestingly, the corresponding velocity profile exhibits a velocity deficit from $\mathrm{y} / \delta=0.1$ to 0.7 and a weak inflexion at $\mathrm{y} / \delta=0.3$. The velocity deficit is the result of a vortex which has moved out of the field of view. The inflexion is caused by the weak remnant of an upstream vortex branch, which is vaguely visible at $\mathrm{y} / \delta=0.35$.

At $t=\mathrm{T} 2$, the vortical structure crosses the $\mathrm{x}-\mathrm{y}$ plane at $\mathrm{x} / D_{o}=3$. The corresponding profile shows a large velocity deficit from $\mathrm{y} / \delta=0.1$ to 0.74 . The velocity deficit is pro-

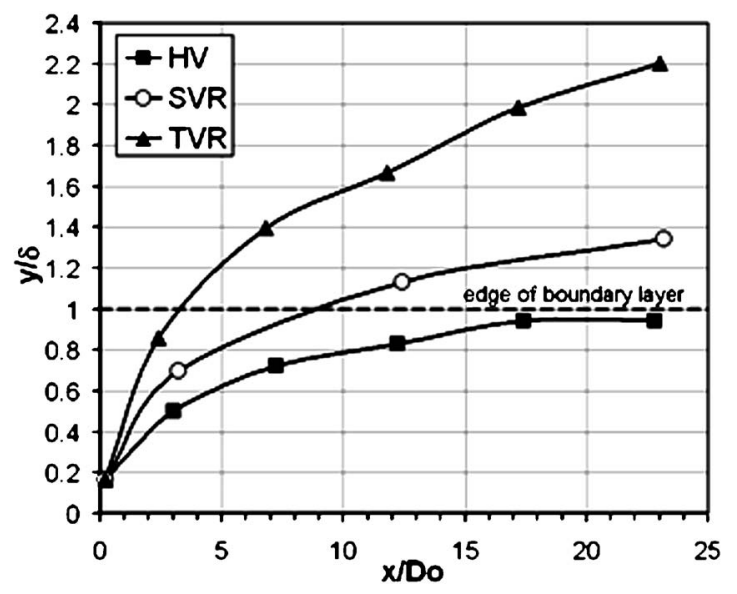

FIG. 7. Trajectory of synthetic jets in the boundary layer (HV=hairpin vortex, $\mathrm{SVR}=$ stretched vortex ring, and $\mathrm{TVR}=$ tilted vortex ring). duced by the upwash induced collectively by the vortex head (i.e., the downstream branch of the vortex) and the newly forming, counter-rotating legs, resulting in low momentum fluid being lifted away from wall. The upwash of fluid is also confirmed in the direction of the velocity vectors. (Note that from hereon "upwash" refers to the movement of fluid away from the wall and "downwash" the movement of fluid toward the wall.) The upwash associated with a hairpin vortex is illustrated schematically in Fig. 9.

Between $\mathrm{y} / \delta=0.74$ and 0.9 , the profile exhibits a small velocity overshoot relative to the undisturbed boundary layer. This overshoot corresponds to the region close to the downstream branch of the vortical structure at $\mathrm{x} / D_{o}=3$. Since the downstream branch has a clockwise rotation, an addition of the vortex tangential velocity with the local boundary layer velocity is expected, resulting in an overshoot. The continued presence of a velocity deficit is observed at $t=\mathrm{T} 3$ between $\mathrm{y} / \delta=0.1$ to 0.8 . However, the deficit is smaller than that at $t=T 2$ since most of the structure has passed through the $\mathrm{x} / D_{o}=3$ plane. By $t=\mathrm{T} 4$ the velocity deficit in the profile has significantly reduced, since the vortical structure has completely passed through the $\mathrm{x} / D_{o}=3$ plane.

Figure 10 shows the phase-averaged boundary layer velocity profiles for a passing stretched vortex ring. There are fewer vortical structures in the field of view compared to the previous case, due to the use of a lower actuation frequency for this structure (Table II). The highly asymmetric rollup of the stretched vortex ring is captured by PIV. The upstream branch of the structure is clearer weaker than the downstream branch and is further weakened by the resident vorticity in the boundary layer. The behavior of the velocity profiles is not too dissimilar to that observed for the hairpin vortices. The passing of the vortical structure across the $\mathrm{x} / D_{o}=3$ plane at $t=\mathrm{T} 2$ and $t=\mathrm{T} 3$ creates a velocity deficit from $\mathrm{y} / \delta=0.1$ to 0.8 and $\mathrm{y} / \delta=0.1$ to 1 , respectively. This velocity deficit is clearly larger and more sustained than that 

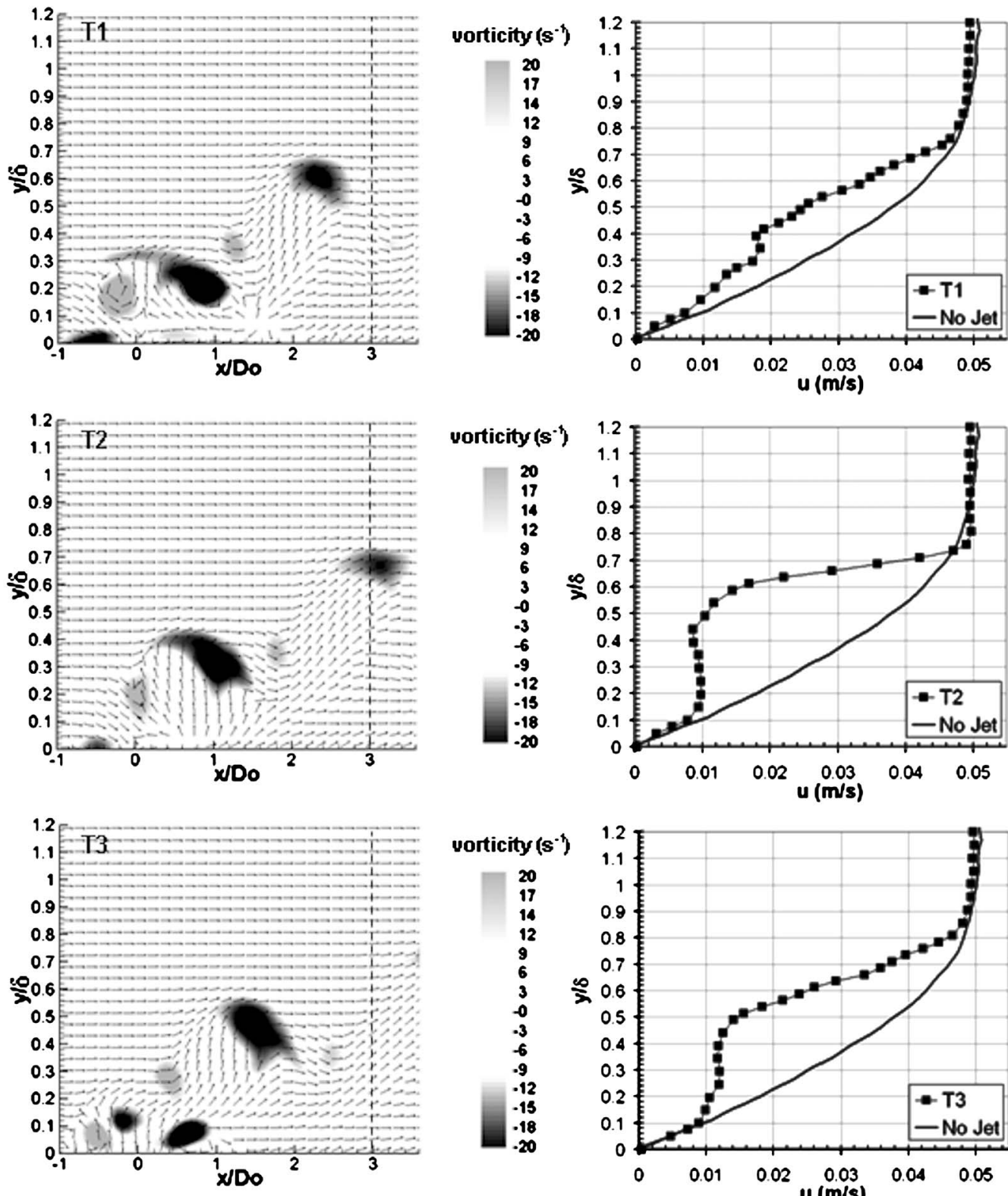

vorticity $\left(\mathrm{s}^{-5}\right)$
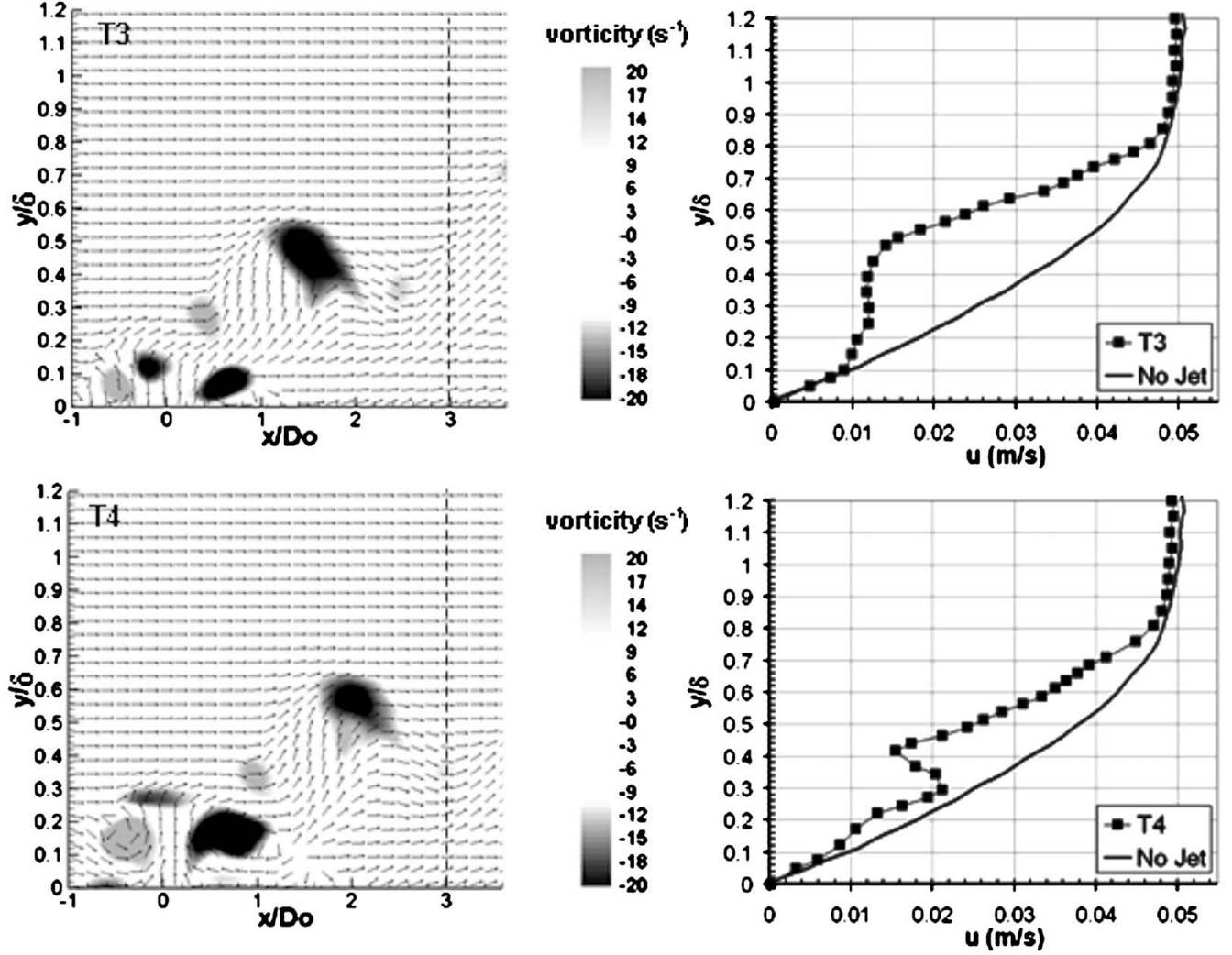

FIG. 8. Transient variation of the centerline phase-averaged boundary layer velocity profile at $\mathrm{x} / D_{o}=3$ for a hairpin vortex $(\mathrm{T} 1=0.63 \mathrm{~T}, \mathrm{~T} 2=0.92 \mathrm{~T}$, $\mathrm{T} 3=0.25 \mathrm{~T}$, and $\mathrm{T} 4=0.50 \mathrm{~T})$.

produced by hairpin vortices, due to the relatively higher strength of the stretched vortex rings marked by a higher $\operatorname{Re}_{L}$. This larger upwash is also captured in the velocity vectors that form an extended zone trailing behind the vortex head.
Figure 11 shows the phase-averaged boundary layer velocity profiles for a passing tilted vortex ring. The vortex in the field of view exhibits a high degree of anticlockwise tilting, in agreement with qualitative observations. ${ }^{15,16} \mathrm{Com}-$ pared to the rollup of hairpin vortices and stretched vortex 


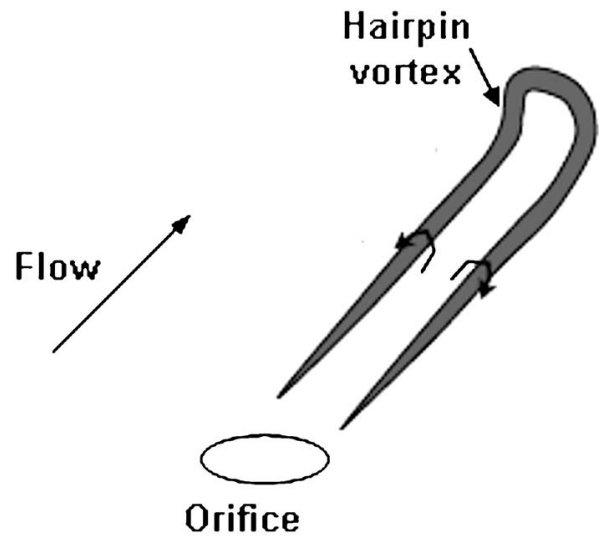

FIG. 9. Schematic of the fluid upwash induced by the head and counterrotating legs of a hairpin vortex.

rings, the tilted vortex ring is more symmetrical. This is a result of the relatively high jet-to-free stream velocity ratio at which this structure is generated, making the upstream branch more resistant to the resident boundary layer.

At the first arbitrarily chosen phase point $t=\mathrm{T} 1$, the vortical structure is approaching the $\mathrm{x} / D_{o}=3$ plane. Despite this, the velocity profile has an observable inflexion at $\mathrm{y} / \delta=0.2$, from which a deficit region extends to $\mathrm{y} / \delta=0.9$. Both the inflexion and velocity deficit are a result of a pair of secondary vortices trailing behind a primary vortex ring that were formed in the previous cycle. As shown from the flow visualization of this structure [Fig. 6(c)], a tilted vortex ring is composed of a leading primary vortex ring and a trailing pair of counter-rotating secondary vortices. The presence of the trailing vortices is evident by the direction of the velocity vectors that trail the primary structure in view in Fig. 11. This region is representative of the upwash induced between the secondary vortices and is responsible for the velocity deficit.

As the downstream branch of the primary vortex ring crosses the $\mathrm{x} / D_{o}=3$ plane at $t=\mathrm{T} 2$, the magnitude of the deficit increases significantly. The upwash induced by the primary ring is responsible for the large velocity deficit between $\mathrm{y} / \delta=0.8$ and 1.06 . The velocity deficit in the region between $\mathrm{y} / \delta=0.2$ to 0.8 is due to the upwash induced by the trailing secondary vortices. Both primary and secondary components of the tilted vortex ring induce an upwash indicating that the trailing secondary vortices have the same sense of rotation as the streamwise components of the primary vortex ring, on each side of the centerline. By $t=\mathrm{T} 4$, the velocity deficit associated with the primary vortex ring has diminished. There is a velocity overshoot between $\mathrm{y} / \delta$ $=0.8$ and 1.1. This overshoot corresponds to the region close to the upstream branch of the vortical structure at $\mathrm{x} / D_{o}=3$. Since the upstream branch has an anticlockwise rotation, an addition of the tangential velocity of the vortex with the local boundary layer velocity is expected, resulting in an overshoot.

It is interesting to observe that at $t=\mathrm{T} 1$ and in particular at $t=\mathrm{T} 2$, a higher velocity gradient than that of the undisturbed profile is generated. At first view, this appears surprising since the trailing vortices induce an upwash, which is conducive of a lower velocity gradient along the centerline. For both hairpin vortices and stretched vortex rings, the near wall velocity gradients along the centerline are smaller than that of the undisturbed profile due to the upwash induced by the counter-rotating legs. By Reynolds analogy, this behavior was observed to correlate with the respective thermal footprints [Figs. 6(a) and 6(b)] since a region of low heat transfer was generated along the centerline. Thus, the higher velocity gradient generated by the tilted vortex rings is conducive to a higher rate of heat transfer, as observed in the corresponding thermal footprint [Fig. 6(c)].

Based on these thermal footprints, it is hypothesized that the trailing secondary vortices induce a tertiary vortex pair with an opposite sense of rotation which, therefore, induces downwash toward the wall. To examine this hypothesis, the results of a numerical simulation for the same flow condition were used. ${ }^{24}$ Figure 12 shows the result of the simulation in which the velocity vector map is superimposed onto the streamwise vorticity contour on a spanwise plane at $\mathrm{x} / D_{o}=3$. The velocity vector map confirms that a tertiary structure is present in the vicinity of the wall at $\mathrm{y} / \delta=0.08$. Since the inclination of the trailing secondary vortices to the wall increases further downstream, it is expected that the strength and presence of the tertiary vortex pair would diminish. For clarification, a schematic diagram of the titled vortex ring and its associated structures is shown in Fig. 13.

\section{Long time-averaged boundary layer profiles}

In order to quantify the lateral extent to which the synthetic jet structures influence the boundary layer, it is necessary to analyze the off-center, as well as the centerline, velocity profiles. An understanding of the changes in the dynamic state of the boundary layer instigated across the span of a passing synthetic jet will help to assess the fluid mixing capability of each synthetic jet structure. Figures 14-16 show the time-averaged impact of the synthetic jet structures on the velocity profiles at $\mathrm{x} / D_{o}=3$ and 24 . For both streamwise locations, off-center velocity profiles are presented for one side of the orifice centerline only since the synthetic jet flow was found to be almost symmetric about the centerline for all cases. For clarity, only profiles along the planes $\mathrm{z} / D_{o}=0,4,8$ and 12 are presented.

The movement of hairpin vortices along the wall creates strong inflexional profiles about the centerline region $\left(\mathrm{z} / D_{o}\right.$ $=0)$ at $\mathrm{y} / \delta=0.1$. This is due to the strong mutual induction of fluid between the hairpin head and counter-rotating legs, which creates large velocity deficits from $\mathrm{y} / \delta \approx 0.12$ to 0.9 . The observations coincide with the findings from the phaseaveraged profiles and as such, this region gives rise to the low heat transfer (light/blue region) generated along the centerline in the corresponding thermal footprint [Fig. 6(a)]. Further outboard $\left(\mathrm{z} / D_{o}=0.4\right)$, there is a noticeable weakening of the inflexion in the profile accompanied by a reduction and movement of the velocity deficit from the wall to the outer part of the boundary layer. For the reduction and movement of this deficit there is a simultaneous filling out of the profile in the near wall region. This contrasting behavior between the inner and outer parts of the profile corresponds to 

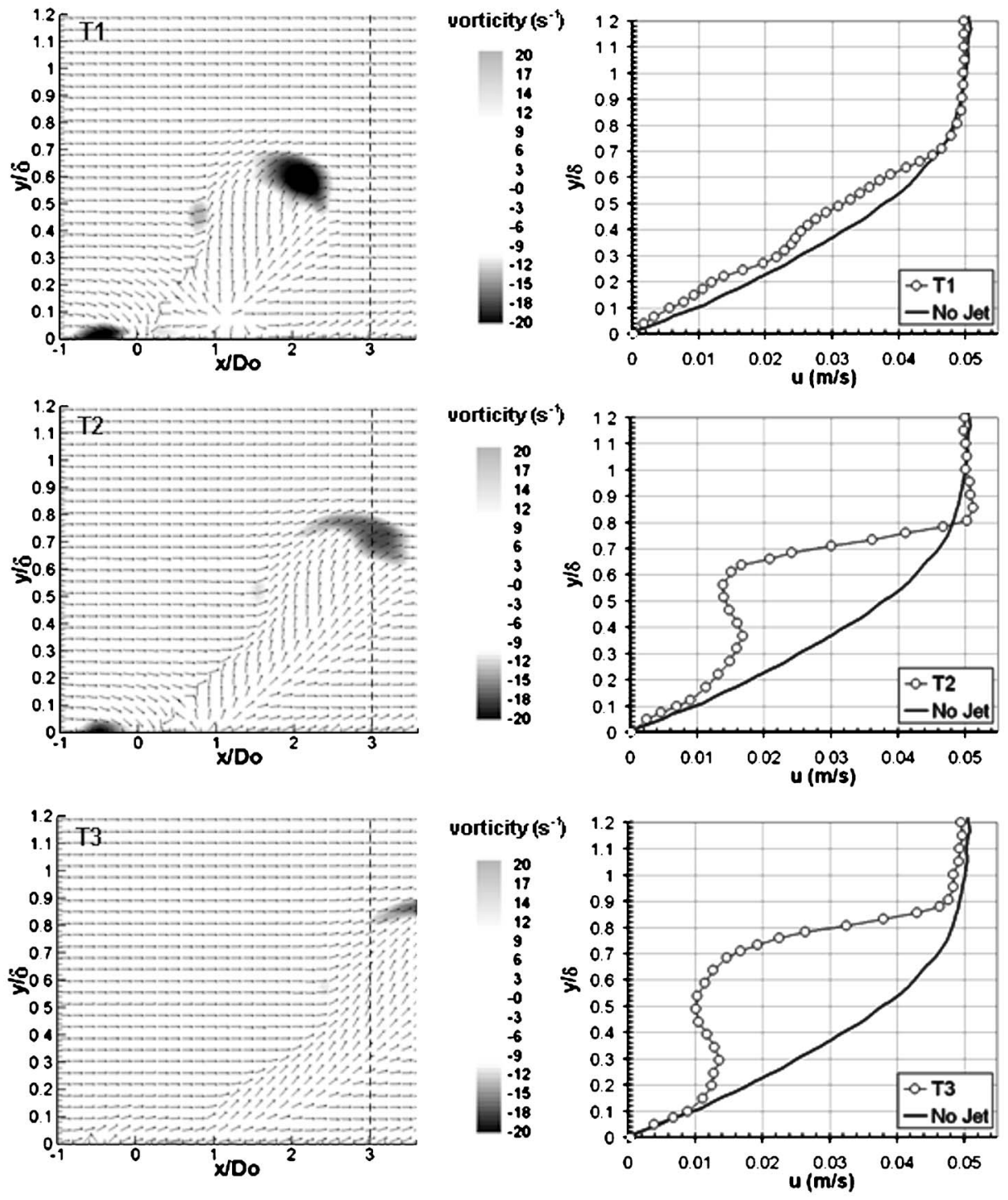

FG. 10. Transient variation of the centerline phase-averaged boundary layer velocity profile at $\mathrm{x} / D_{o}=3$ for a stretched vortex ring $(\mathrm{T} 1=0.71 \mathrm{~T}$, $\mathrm{T} 2=0.88 \mathrm{~T}, \quad \mathrm{~T} 3=0.04 \mathrm{~T}$, and $\mathrm{T} 4$ $=0.17 \mathrm{~T})$.
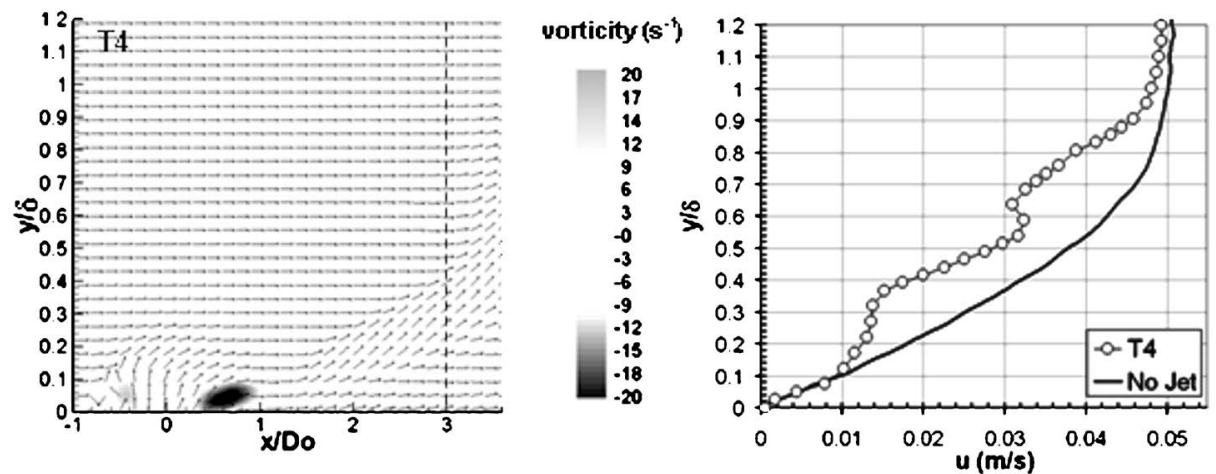

the redistribution of momentum across the boundary layer. That is, the mixing of low momentum fluid near the wall with higher momentum fluid away from the wall. As a result, a local maximum in the near wall velocity gradient is attained at approximately $\mathrm{z} / D_{o}=0.4$. It is believed this lateral position coincides with a location just outboard of the hairpin leg, in which there is a maximum downwash. This is conducive of the increased heat transfer produced outboard of the centerline in the corresponding thermal footprint [Fig. 6(a)]. The profile further outboard $\left(\mathrm{z} / D_{o}=1.2\right)$ is more relaxed with smaller a velocity deficit in the outer region. There is also a noticeable reduction in the near wall velocity gradient, signifying a reduction of the lateral effect of the synthetic jet.

A similar lateral trend in the velocity profiles further downstream at $\mathrm{x} / D_{o}=24$ is observed [Fig. 14(b)]. The profiles are clearly more relaxed than those upstream, reflecting the reducing strength of the hairpin vortices due to streamwise dissipation in the boundary layer. Profiles at $\mathrm{z} / D_{o}=0$ and 0.4 consist of significant velocity deficits, evident of the continued upwash generated between the counter-rotating 

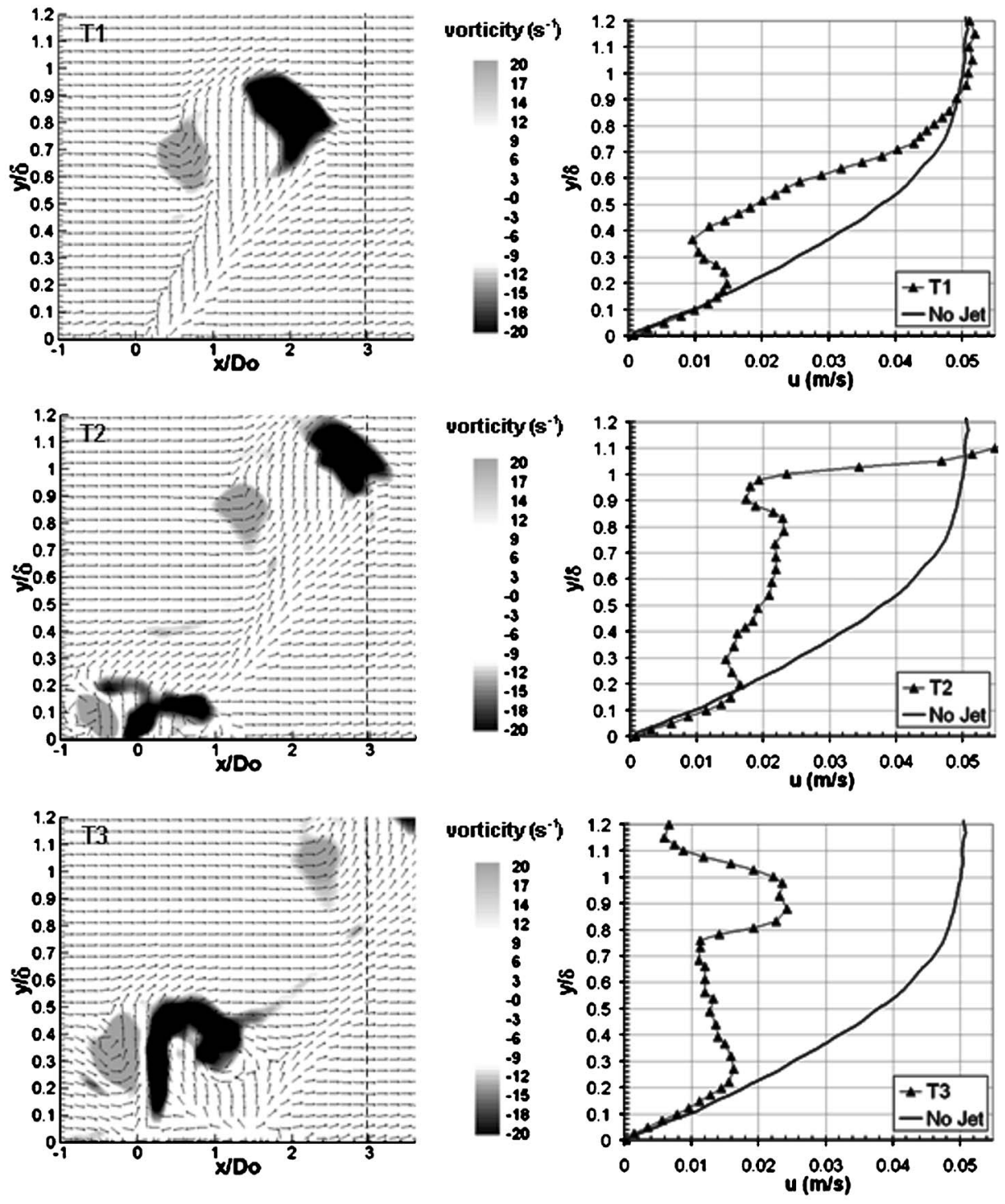

FIG. 11. Transient variation of the centerline phase-averaged boundary layer velocity profile at $\mathrm{x} / D_{o}=3$ for a tilted vortex ring $(\mathrm{T} 1=0.00 \mathrm{~T}, \mathrm{~T} 2$ $=0.25 \mathrm{~T}, \quad \mathrm{~T} 3=0.54 \mathrm{~T}, \quad$ and $\mathrm{T} 4$ $=0.75 \mathrm{~T}$ ).
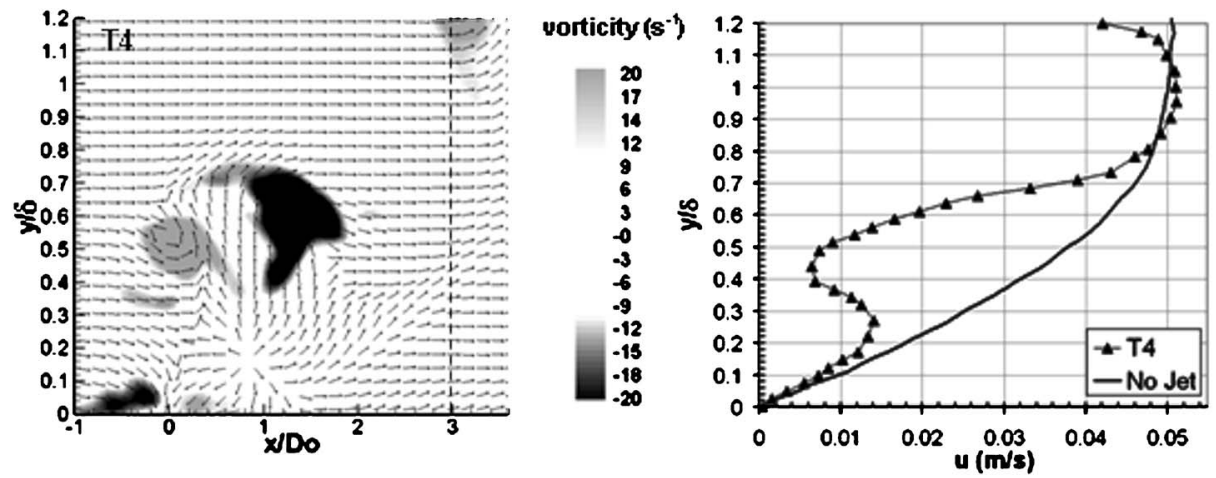

legs. Movement outboard of the centerline gives rise to fuller profiles with a corresponding increase in the near wall velocity gradient. A maximum in the gradient occurs at $\mathrm{z} / D_{o} \approx 1.2$.

The profiles influenced about the centerline region $\left(\mathrm{z} / D_{o}=0\right)$ by stretched vortex rings at $\mathrm{x} / D_{o}=3$ [Fig. 15(a)] exhibit a velocity deficit between $\mathrm{y} / \delta=0.1$ and 0.8 . In the time-averaged sense, this velocity deficit is less severe than that produced by the hairpin vortices, although the stretched vortex rings generate a greater deficit and hence fluid lift-up at a given phase point on account of their greater strength. It is believed that due to their higher periodicity in the near field of the orifice, the hairpin vortices, despite their relatively lower strength, induce a consistently higher level of fluid lift up across a cycle. Peaks in the velocity gradients are observed at $\mathrm{z} / D_{o}=0.4$ in the near field and at $\mathrm{z} / D_{o}=0.8$ in the far field [Fig. 15(b)].

The occurrence of off-center maximas in the near wall velocity gradient is consistent with the outboard downwash produced by the presence of counter-rotating legs. However, 


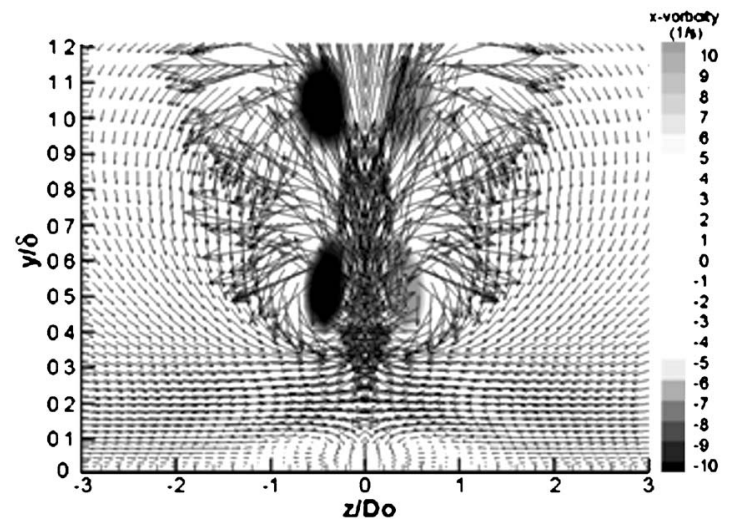

FIG. 12. Numerical simulation of a tilted vortex ring across a spanwise plane at $\mathrm{x} / D_{o}=3$.

relative to the hairpin vortices, the stretched vortex rings are seen to produce fuller profiles across the span of the jet at $\mathrm{x} / D_{o}=24$. This indicates that the stretched vortex rings have a greater fluid mixing ability in redistributing higher momentum fluid from the outer part of the boundary layer to the near wall region. This is inherently due to greater vortex strength and cross flow penetration into the boundary layer. Figure 16(a) shows the time-averaged boundary layer velocity profiles influenced by tilted vortex rings at $\mathrm{x} / D_{o}=3$. The lateral trend of the profiles differs to those for hairpin vortices and stretched vortex rings at the same streamwise location. There is a maximum in the near wall velocity gradient along the centerline, corresponding to the downwash induced by the tertiary vortex in the vicinity of the wall. This observation correlates with the centerline region of high heat transfer in the thermal footprint [Fig. 6(c)]. This maximum gradient is also greater than those observed for the other cases at different lateral positions, indicating that the greatest level of fluid mixing occurs along the centerline of the tilted vortex ring flow in the near field region. This enhancement of near wall fluid mixing is accompanied by significant momentum loss in the outer part of the boundary layer marked by the large velocity deficit, which originates at $\mathrm{y} / \delta \approx 0.2$. This deficit is due to the primary vortex ring and trailing secondary vortices. Further outboard $\left(\mathrm{z} / D_{o}=0.2\right)$, there is a reduction in the velocity gradient corresponding to movement away from the region of maximum downwash induced between the tertiary vortex pair. The velocity deficit originating at $\mathrm{y} / \delta \approx 0.2$ also reduces. This reduction coincides with movement from the inboard side of a trailing secondary vor-
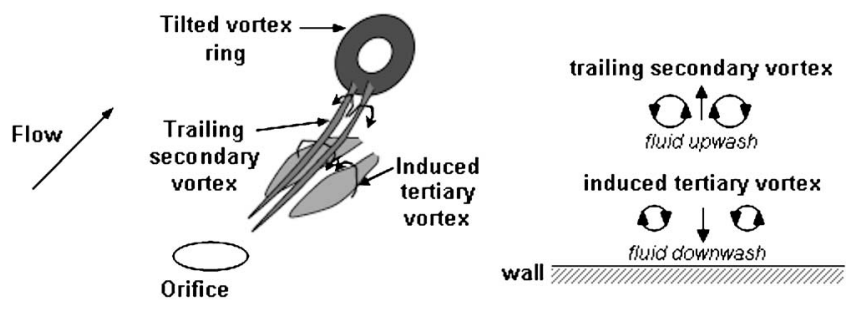

FIG. 13. Schematic of the secondary and tertiary vortical structures associated with a tilted vortex ring.
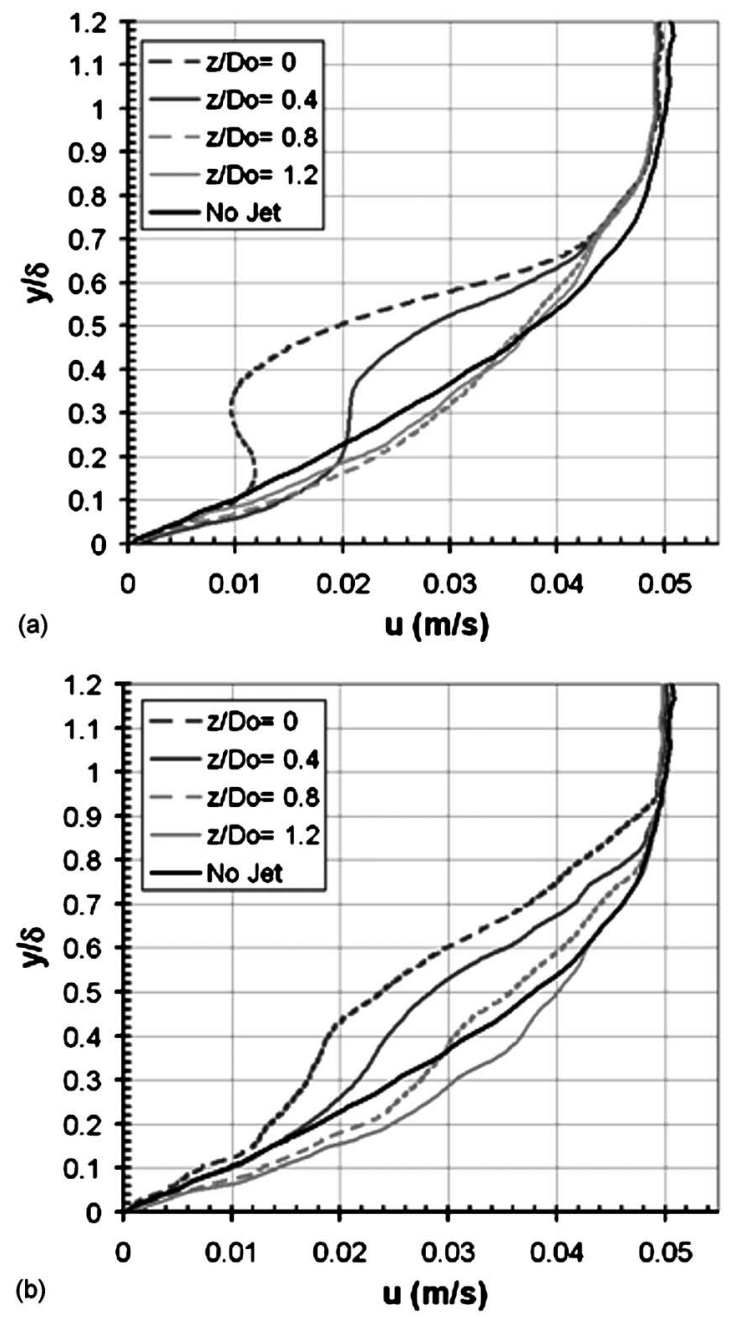

FIG. 14. Long time-averaged boundary layer velocity profiles for hairpin vortices at (a) $\mathrm{x} / D_{o}=3$ and (b) $\mathrm{x} / D_{o}=24$.

tex, which induces a flow away from the wall to the outboard side, which directs the local flow toward the wall.

Further downstream at $\mathrm{x} / D_{o}=24$ [Fig. 16(b)], all of the profiles have become fuller and more relaxed compared to those upstream. It is apparent that the near wall velocity gradient of the profiles in the centerline region $\left(\mathrm{z} / D_{o}=0\right.$ and $0.4)$ have reduced relative to those upstream. This is indicative of the propagation of the secondary vortex pair away from the wall, resulting in a weaker induced tertiary vortex. With increase in lateral distance from the centerline, both the near wall velocity gradients and the deficits in the outer part of the profiles reduce.

\section{POTENTIAL OF SYNTHETIC JETS FOR FLOW SEPARATION CONTROL}

\section{A. Long time-averaged wall shear stress distributions}

The spanwise influence of each synthetic jet vortical strucuture on the wall shear stress distribution in the near field $\left(\mathrm{x} / D_{o}=3\right)$ and far field $\left(\mathrm{x} / D_{o}=24\right)$ of the orifice are shown in Figs. 17(a) and 17(b), respectively. For this analysis, the streamwise components of wall shear stress, as shown in Fig. 3, were used. Note that at both streamwise 

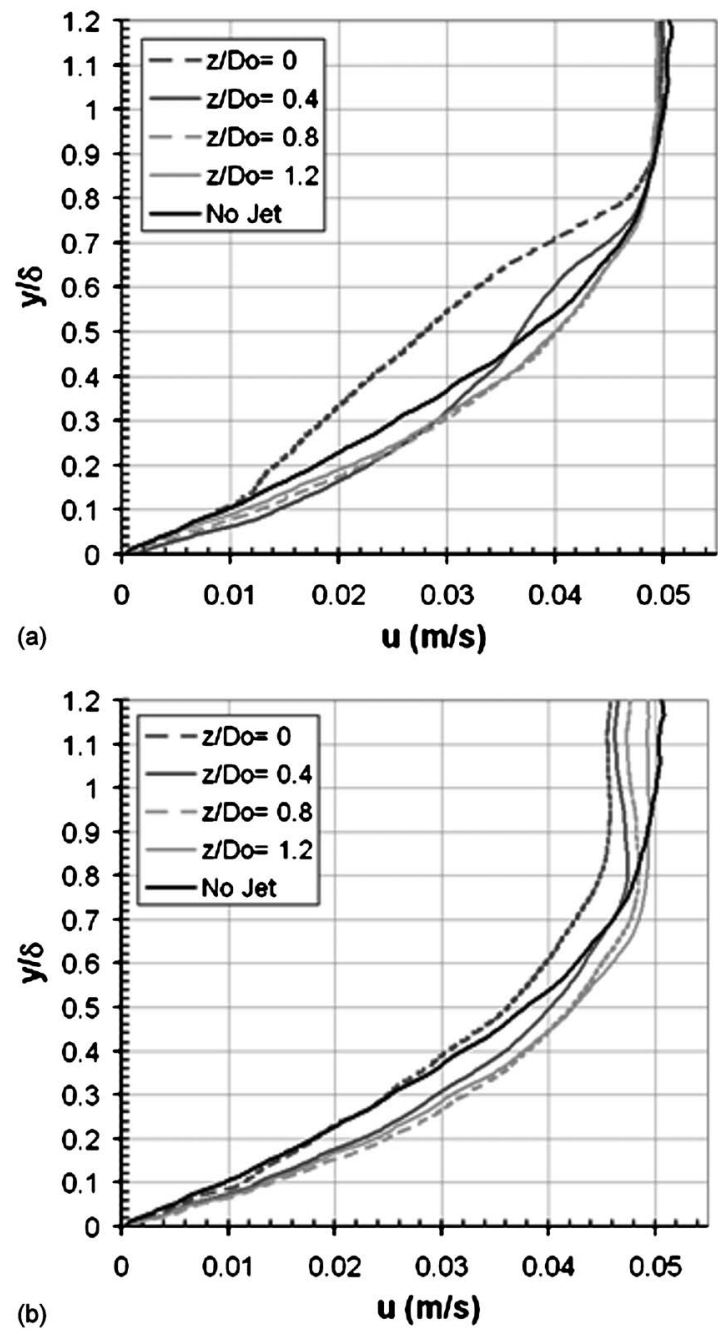

FIG. 15. Long time-averaged boundary layer velocity profiles for stretched vortex rings at (a) $\mathrm{x} / D_{o}=3$ and (b) $\mathrm{x} / D_{o}=24$.

locations, the wall shear stress is normalized by the local undisturbed value, $\tau_{o}$. The level of the undisturbed value is indicated by the dash line.

At $\mathrm{x} / D_{o}=3$, both hairpin vortices and stretched vortex rings produce similar spanwise trends of wall shear stress distribution. In both cases, two off-center peaks in wall shear stress are generated almost symmetrically about the centerline region. Wall shear stress reduces toward the centerline region and outboard of the off-center peaks in both cases.

For the hairpin vortices, the off-center peak in wall shear stress located at $\mathrm{z} / D_{o}=0.4$ coincides with a maximum in the near wall velocity gradient at the same location, as previously identified from Fig. 14(a). Similarly, the near wall velocity gradients of the off-center profiles reduce further outboard, coinciding with the trend of reducing wall shear stress. This trend is also mirrored on the other side of the centerline, where a peak in wall shear stress is generated at $\mathrm{z} / D_{o}=-0.6$. The level of wall shear stress increase at these peaks is approximately $70 \%-75 \%$ above the undisturbed value.

Likewise, the peak wall shear stress produced by the stretched vortex rings at $\mathrm{z} / D_{o}=0.4$ coincides with the maxi-
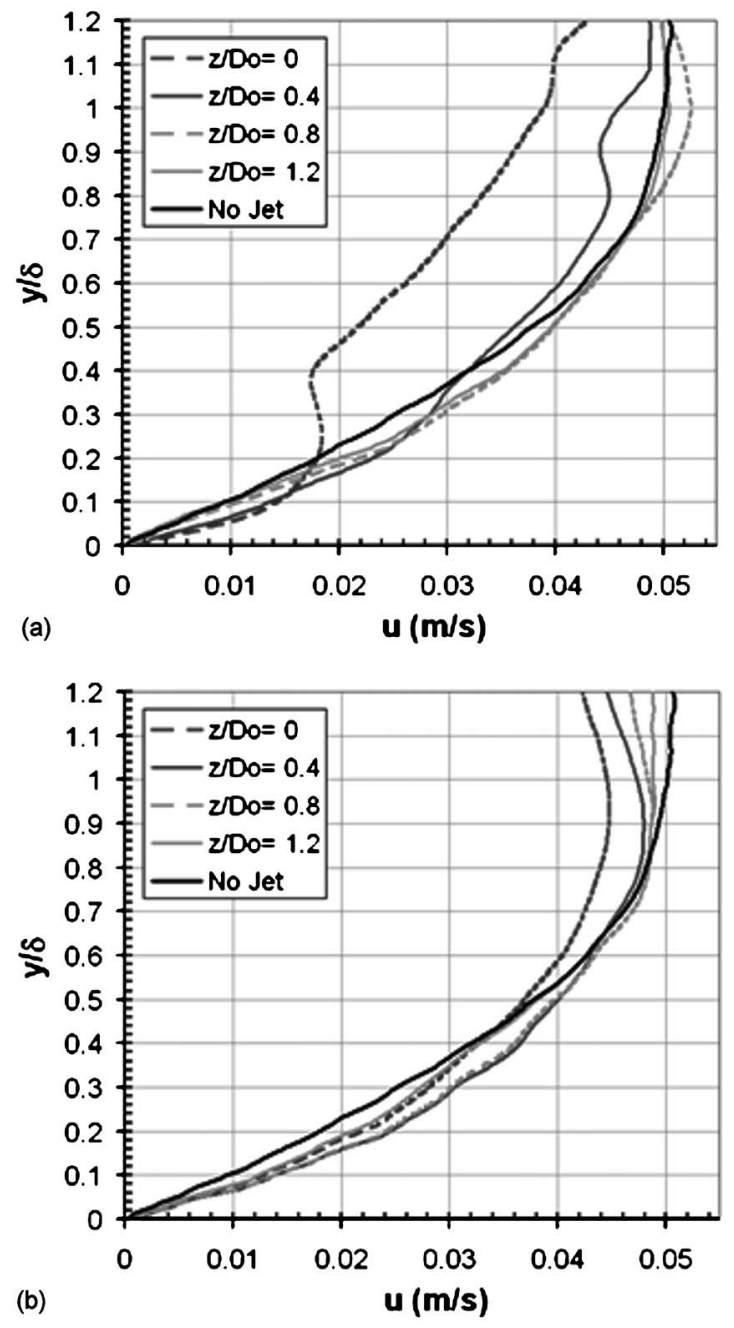

FIG. 16. Long time-averaged boundary layer velocity profiles for tilted vortex rings at (a) $\mathrm{x} / D_{o}=3$ and (b) $\mathrm{x} / D_{o}=24$.

mum near wall velocity gradient of the profile at the same spanwise location [Fig. 15(a)]. The other peak is located at $\mathrm{z} / D_{o}=-0.6$. Perturbation of the undisturbed wall shear stress by the stretched vortex rings produces a maximum increase of $60 \%-65 \%$ and is, therefore, slightly less than the increase produced by the hairpin vortices at the same streamwise location. In both cases, the off-center peaks in wall shear stress correspond to spanwise positions outboard of each counterrotating leg where there is a region of induced downwash.

The trend in wall shear stress produced by the tilted vortex rings at $\mathrm{x} / D_{o}=3$ is markedly different. In this case, there is only a single peak in wall shear stress that is generated in the centerline region $\left(\mathrm{z} / D_{o}=-0.2\right)$ with shear stress decreasing laterally on either side of the peak. Similarly, this reduction coincides with a reducing trend in the near wall velocity gradients of the profiles with spanwise distance from the centerline [Fig. 16(a)]. The enhancement of wall shear stress at the centerline peak relative to the undisturbed value is approximately $90 \%$, which is greater than the other two cases. The peak in wall shear stress is associated with the downwash induced between the tertiary vortex pair.

One reason as to why the tertiary vortex pair generates a higher peak in wall shear stress relative to both hairpin vor- 

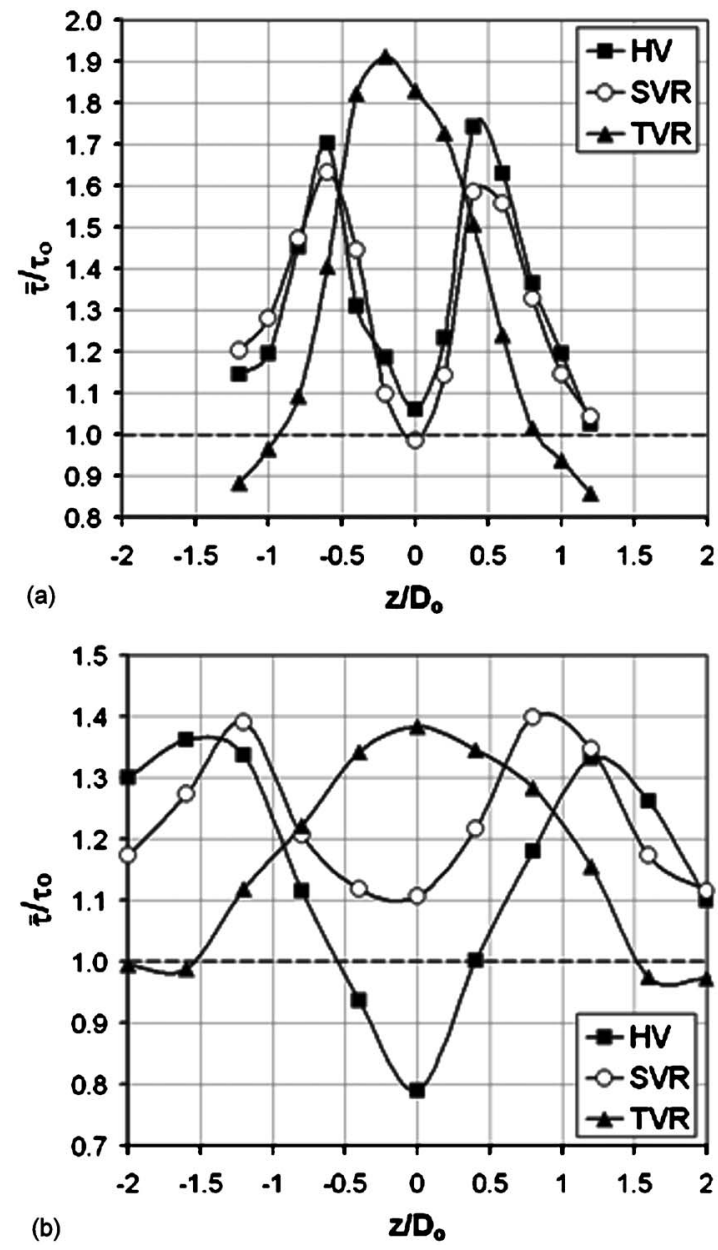

FIG. 17. The effect of synthetic jets on the spanwise wall shear stress distribution at (a) $\mathrm{x} / D_{o}=3$ and (b) $\mathrm{x} / D_{o}=24(\mathrm{HV}=$ hairpin vortex, SVR =stretched vortex ring, and TVR=tilted vortex ring; dashed line corresponds to the undisturbed value).

tices and stretched vortex rings may be due to its sense of rotation. Experiments of different longitudinal vortex pair configurations embedded in a zero-pressure gradient turbulent boundary layer were conducted ${ }^{19}$ in which vortex configurations included counter-rotating vortices with common flow up (upwash) and common flow down (downwash). It was found that vortices in the common flow down configuration have little interaction with each other, but a much stronger interaction with the viscous flow near the wall. Conversely, vortices in the common flow up configuration interacted strongly with each other and in doing so, convect each other away from the wall. Spanwise pertubations in the skin friction measurements showed a larger gain in the common flow down case than in the common flow up case. In the time-averaged sense, the behavior of hairpin vortices and stretched vortex rings is akin to the common flow up configuration and that of the tertiary vortex pair is similar to the common flow down configuration.

At $\mathrm{x} / D_{o}=24$ [Fig. 17(b)], the pertubations of wall shear stress by hairpin vortices and stretched vortex rings exhibit similar trends to those at $\mathrm{x} / D_{o}=3$. Both structures generate off-center peaks in wall shear stress, albeit at a smaller magnitude to those upstream due to vortex decay in the boundary layer. Similarly, the positions of the peaks correspond to maximas attained in the velocity gradients of the profiles. Both sets of peaks in wall shear stress for the two structures have moved laterally outboard of those further upstream, indicating a modest increase in spanwise penetration into the boundary layer.

There are also some notable differences in the trends of wall shear stress between both hairpins and stretched vortex rings. Contrary to the observation made in the near field location, the stretched vortex rings generate slightly larger peaks in wall shear stress at $\mathrm{x} / D_{o}=24$. The enhancement in wall shear due to the stretched vortex rings is approximately $40 \%$ compared to $35 \%$ for the hairpin vortices (although this difference is not critical given the uncertainty in the measurements, as detailed in Sec. II A). Coupled with this, the stretched vortex rings also generate a greater centerline value of wall shear stress, despite having an almost identical value as that exhibited by the hairpins further upstream. These differences are largely attributed to differences in vortex strength and vortex location in the boundary layer. A relatively greater vortex strength coupled with a jet trajectory that runs just beyond the edge of the boundary layer (Fig. 7), ensures that the stretched vortex rings entrain higher momentum fluid from the outer part of the boundary layer to the wall. This interchange results in a stronger downwash outboard of the legs that generates a higher increase in wall shear stress. The smaller minimum of centerline wall shear stress pertaining to the hairpin vortices is by virtue of their counter-rotating legs being embedded closer to the wall hence inducing a larger upwash. This upwash is replaced with an outboard downwash of lower momentum fluid relative to that of the stretched vortex rings, due to their location in the boundary layer.

For the spanwise distribution of wall shear stress associated with the tilted vortex rings at $\mathrm{x} / D_{o}=24$, a peak in wall shear continues to be produced along the centerline, albeit smaller than the upstream peak. It is therefore evident that the tertiary vortex pair continues to bring fluid toward the wall along the centerline, resulting in a peak wall shear stress enhancement of almost $40 \%$ relative to the undisturbed value. However, this is significantly reduced compared to an enhancement of $90 \%$ at $\mathrm{x} / D_{o}=3$ and represents the largest reduction in peak wall shear stress enhancement of all three structures. This reduction in wall shear stress enhancement coincides with the weakening of the induced tertiary vortex pair due to the increasing propagation of the trailing secondary vortices away from the wall with streamwise distance. Incidentally, the stretched vortex rings exhibit the smallest reduction in peak wall shear stress between the near field $(65 \%)$ and far field (40\%) regions, i.e., a maximum reduction of $25 \%$. For hairpin vortices, the maximum reduction is approximately $40 \%$.

The wall shear stress distribution relative to each synthetic jet vortical structure in Fig. 17 varies spatially in the spanwise direction. To assess the relative effectiveness of each vortical structure, it is useful to consider the net spanwise enhancement of wall shear stress at each streamwise location. This is obtained by evaluating the spatial averages of the wall shear stress distributions. 
TABLE IV. Normalized spanwise-mean wall shear stress enhancement and net reductions of synthetic jets between the near field and far field.

\begin{tabular}{lccc}
\hline \hline & \multicolumn{3}{c}{$\begin{array}{c}\bar{\tau} / \tau_{o} \\
(\%)\end{array}$} \\
\cline { 2 - 3 } Structure type & $\mathrm{x} / D_{o}=3$ & $\mathrm{x} / D_{o}=24$ & $\begin{array}{c}\Delta \bar{\tau} / \tau_{o} \\
(\%)\end{array}$ \\
\hline Hairpin vortex & 33 & 16 & -17 \\
Stretched vortex ring & 30 & 23 & -7 \\
Tilted vortex ring & 32 & 16 & -16 \\
\hline \hline
\end{tabular}

The net reduction in wall shear stress between the near field and far field locations will also provide an indication of the vortex persistence in the boundary layer. The streamwise persistency of the vortical structure is an important attribute for the effectiveness of synthetic jets in a boundary layer. For a given power consumption, the structure produced by the synthetic jet will be more desirable for flow control purposes if it is capable of maintaining its strength and hence its ability to redistribute momentum across the boundary layer over a longer distance downstream. Table IV shows values of the normalized, spanwise-mean wall shear stress enhancement pertaining to each vortical structure at $\mathrm{x} / D_{o}=3$ and 24 and the respective net reductions between each location.

At $\mathrm{x} / D_{o}=3$, the spanwise-mean values show that the net increase of wall shear stress for each case is almost identical, varying slightly between $30 \%$ and $33 \%$. This is particularly interesting as the peaks in wall shear stress enhancement vary significantly from $60 \%$ to $90 \%$, according to Fig. 17(a). Most noteworthy is the difference between the peak (90\%) and spanwise-mean (32\%) enhancement of wall shear stress associated with the tilted vortex rings. In this case, the increase in wall shear stress is highly localized along the centerline and is therefore laterally limited.

The differences in spanwise-mean wall shear stress enhancement between each case are prominent in the far field location. At $\mathrm{x} / D_{o}=24$, the stretched vortex rings generate the largest net increase in wall shear stress at 23\%. Both hairpin vortices and tilted vortex rings (tertiary vortex pair) generate a $16 \%$ net increase in wall shear stress. Therefore, stretched vortex rings exhibit the smallest reduction in spanwise-mean wall shear stress enhancement between the near and far field locations. The corresponding reduction $(-7 \%)$ is approximately half that for hairpin vortices $(-17 \%)$ and tilted vortex rings $(-16 \%)$, indicating that stretched vortex rings are the most persistent synthetic jet vortices in the boundary layer.

The persistency of a vortical structure is linked to its strength and location in the boundary layer. Due to its higher $\mathrm{Re}_{L}$, the strength of the stretched vortex rings is larger than both the hairpin vortices and the induced tertiary vortex pair associated with tilted vortex rings. Second, the stretched vortex rings are located further away from the wall compared to the aforementioned structures. The hairpin vortices and in particular the tertiary vortex pair are both embedded close to the wall. This is conducive to a faster rate of streamwise vortex decay due to viscous dissipation, thereby reducing the persistency of those vortices.
TABLE V. Normalized spatial and temporal rms wall shear stress variatons produced by synthetic jets.

\begin{tabular}{|c|c|c|c|c|}
\hline \multirow[b]{2}{*}{ Structure type } & \multicolumn{2}{|c|}{$\begin{array}{c}\text { Spatial } \underset{(\%)}{\mathrm{rms}}, \tau_{\mathrm{rms}} / \tau_{\mathrm{av}} \\
\end{array}$} & \multicolumn{2}{|c|}{$\begin{array}{c}\text { Temporal } \mathrm{rms}, \tau_{\mathrm{rms}} / \tau_{\mathrm{av}} \\
(\%)\end{array}$} \\
\hline & $\mathrm{x} / D_{o}=3$ & $\mathrm{x} / D_{o}=24$ & $\mathrm{x} / D_{o}=3$ & $\mathrm{x} / D_{o}=24$ \\
\hline Hairpin vortex & 17 & 15 & 3 & 2 \\
\hline Stretched vortex ring & 16 & 8 & 20 & 2 \\
\hline Tilted vortex ring & 29 & 13 & 22 & 3 \\
\hline
\end{tabular}

\section{B. Rms wall shear stress distributions}

Evaluation of the potential flow control effectiveness can be enhanced by considering two additional parameters-the spatial rms wall shear stress and the temporal rms wall shear stress. The spatial rms wall shear stress can be taken as a measure of the extent of spatial variations in wall shear stress hence resulting in spatial variations in flow control effectiveness. The temporal rms wall shear stress allows assessment of the ability of a synthetic jet in keeping flow separation suppressed within an actuation cycle.

Both the spatial and temporal rms wall shear stress values, $\tau_{\mathrm{rms}}$, are normalized by the local averaged wall shear stress, $\tau_{\text {ave }}$, and are presented in Table V. The normalized spatial rms wall shear stress is relatively high for the titled vortex ring case, especially in the near field with a value of $29 \%$. While values of the normalized spatial rms wall shear stress is similar for the hairpin and stretched vortex ring case at $\mathrm{x} / D_{o}=3$, its magnitude reduces more dramatically in the far field at $\mathrm{x} / D_{o}=24$ for the stretched vortex ring case (i.e., $8 \%$ compared to $15 \%$ for the hairpin vortex case). This results in a much smoother wall shear stress distribution across the span for the stretched vortex ring case, as also shown in Fig. 17(b).

Normalized values of temporal rms wall shear stress were obtained along streamwise planes corresponding to regions of maximum fluid downwash and wall shear stress (that is, off-center planes for the hairpin vortex and stretched vortex ring cases and along the centerline plane for the tilted vortex ring case). Tilted vortex rings and stretched vortex rings have the largest temporal rms wall shear stress in the near field at $22 \%$ and $20 \%$ of the local averaged wall shear stress, respectively. While these values are larger than that for hairpin vortices at the same streamwise location, the far field rms values indicate that the difference between each case is negligible, with variations between $2 \%$ and $3 \%$ of local averaged wall shear stress. Therefore, the ability to suppress flow separation within an actuation cycle is comparable for each case.

\section{Flow control effectiveness}

This study allows for a relative assessment of the potential separation control effectiveness of the typical synthetic jet vortices. This is based on the understanding that the vortical structures produced by a SJA in a laminar flow are similar to those generated in a turbulent one. The latter is a more likely scenario for industrial applications, such as air- 
craft high-lift devices. Although the present study is undertaken in a zero-pressure gradient boundary layer, the basis of assessing the potential flow control effectiveness comes from the ability of the synthetic jet to introduce some measurable change to the flow, e.g., wall shear stress enhancement. Therefore, the knowledge gained from this study should provide a useful indicator of the potential of synthetic jets to delay separation.

Given the definition of an optimal coherent structure for separation control ${ }^{18}$ and the results of this investigation, the stretched vortex rings offer the greatest potential effectiveness for flow separation control. In conjunction with the experimental findings, considerations are given to the practical settings in which the SJA system is likely to be operated, to justify the selection of stretched vortex rings as a candidate flow control structure. These are detailed as follows:

(1) Although the net enhancement of wall shear stress is similar for all the vortical structures in the near field $\left(\mathrm{x} / D_{o}=3\right)$, clear differences emerge in the far field $\left(\mathrm{x} / D_{o}=24\right)$ where the stretched vortex rings produce the largest enhancement of wall shear stress and hence potential to delay separation. The stretched vortex rings are also the most persistent structures in the boundary layer by virtue of producing the smallest streamwise reduction in wall shear stress enhancement. From a practical viewpoint, vortex persistency is particularly important where implementation of SJAs near to the baseline-mean flow separation region is not always feasible. Investigation of a SJA array to delay separation on a deflected aileron ${ }^{25}$ revealed that due to weight and structural issues, the array could not be implemented on the aileron. The array was alternatively implemented upstream of the aileron hinge line.

(2) Figure 17 indicates that both hairpin vortices and tilted vortex rings exhibit regions in the spanwise distributions of wall shear stress that fall below the undisturbed wall shear stress value (specifically along the centerline and off-center, respectively), which is also manifested by a relatively larger spatial rms in the wall shear stress. For incipient flow separation, these regions would be conducive to strong flow reversal occurring between regions of attached flow and would therefore result in a pronounced waviness in the separation line. In contrast, the stretched vortex rings consistently produce an increase in wall shear stress across the span relative to the undisturbed value, which would ultimately result in a much smoother delay in the separation line. To ensure that flow separation is suppressed within an actuation cycle, a low temporal rms wall shear stress is considered to be desirable. Negligible differences in temporal $\mathrm{rms}$ wall shear stress between each case in the far field confirms that the generation of stretched vortex rings would cause the least streamwise variation to a separation line.

(3) The theoretical and measured peak jet velocities for generating a stretched vortex ring is marginally lower compared to the other structures (Table III). As such, a stretched vortex ring also has the lowest jet fluid power. Based on the measured jet velocities, $u_{\text {peak }}$, and for a given fluid density, $\rho$ (water) and orifice area, $A(5 \mathrm{~mm}$ diameter), the maximum fluid power, $W_{F}=\frac{1}{2} \rho u_{\text {peak }}{ }^{3} A$, associated with a hairpin vortex, stretched vortex ring, and tilted vortex ring is $1.0,0.8$, and $5.6 \mu \mathrm{W}$, respectively. The SJA electrical input power, $W_{E}$, required to generate a given fluid output power is equivalent to $W_{E}=W_{F} / \eta$, where $\eta$ is the electric-to-fluidic power conversion efficiency. No measurement of the efficiency was possible from this work. However, for a given SJA diaphragm and internal geometry, the efficiency will depend largely on the orifice losses. It has been shown ${ }^{26-28}$ that the pressure drop and hence power dissipation for oscillatory flow through an orifice primarily scales with the Stokes number, which is a function of frequency. Given the lower actuation frequency for generating stretched vortex rings (Table II), it is likely that the associated power consumption will be comparable to or lower than that for hairpin vortices. Therefore, the practical implications of generating stretched vortex rings may be more desirable in terms of reducing the mass and power consumption of the actuator system. Namely, an array of SJAs for generating stretched vortex rings would require fewer actuators and therefore less flow energy expenditure per unit span to achieve the same wall shear stress gains compared to the generation of the other vortical structures.

In specific comparisons against tilted vortex rings:

(1) For stretched vortex rings, the circulation ejected from the orifice is contained entirely within the vortices. The counter-rotating streamwise legs of these structures are responsible for the enhancement of wall shear stress. Conversely, none of the ejected circulation that is contained in the tilted vortex ring (i.e., the leading vortex ring and trailing secondary vortices) is used directly for wall shear stress enhancement. Rather, only the induced circulation of the tertiary vortex pair is responsible for enhancing wall shear stress. This has implications for the efficient production of structures for flow control. In comparison, stretched vortex rings are more efficient than tilted vortex rings in terms of useful work done by the SJA, i.e., the ratio of ejected circulation to wall shear stress enhancement. Despite having the greater vortex strength, both the leading vortex and trailing secondary vortices of the tilted vortex ring represent energy wasted in terms of potential flow control effectiveness.

(2) The ability to modulate the stretched vortex ring strength is straightforward in comparison to an induced vortex associated with a tilted vortex ring. The key to enhancing vortex strength and ensuring the structure persists in the boundary layer is dependent on a moderate $\operatorname{Re}_{L}$ (trade-off between adequate vortex strength and power consumption) and a $V R$ that permits a vortex trajectory along the edge of the boundary layer. This can be achieved through modulation of the diaphragm oscillatory frequency and displacement, as required in the context of the parameter map. ${ }^{16}$ Conversely, the issue of strength modulation for an induced vortex is more com- 
plex, since it is largely dependent on the strength and location (relative to the wall) of the vortex from which it is induced.

\section{CONCLUSIONS}

PIV measurements of the vortical structures formed by the interaction of synthetic jets with a flat plate laminar boundary layer have been carried out. The purpose of this work was to investigate the impact of the vortical structures on the boundary layer and to identify the structures with potential for flow separation control. The findings from this investigation are summarized as follows:

(1) Phase-averaged vorticity contours at the centerline show that hairpin vortices and stretched vortex rings develop asymmetrically. In both cases, the upstream branch of the vortex is weakened by the countervorticity of the oncoming boundary layer.

(2) Phase-averaged vorticity contours show that tilted vortex rings experience a symmetrical development, with both upstream and downstream branches of the vortex of similar strength. This is a result of the relatively high jet-to-free stream velocity ratio at which this structure is generated, making the upstream branch more resistant to the oncoming boundary layer.

(3) In the time-averaged sense, both hairpin vortices and stretched vortex rings exhibit characteristics akin to a longitudinal vortex pair with a common upflow embedded in a boundary layer. The motion of the streamwise counter-rotating legs induces an inboard upwash with corresponding deficits in the boundary layer profiles and local minimas in wall shear stress. Conversely, a downwash is induced outboard of the legs, producing fuller profiles off-center and local peaks in wall shear stress.

(4) The trailing secondary vortices associated with the tilted vortex rings induce a tertiary vortex pair near the wall. In the time-averaged sense, this vortex pair exhibits characteristics akin to a longitudinal vortex pair with a common downflow, embedded in a boundary layer. As such, the vortices induce a downwash along the centerline, generating a fuller boundary layer profile and a local peak in wall shear stress.

(5) Stretched vortex rings offer greater potential for flow separation control compared to the other vortices in terms of the following:

(i) higher net enhancement of wall shear stress,

(ii) greater streamwise persistency of wall shear stress, and

(iii) lower spatial rms wall shear stress.

Coupled with expectations of a comparable power consumption with the other vortices, the stretched vortex ring may be considered as a candidate flow control structure for SJAs applied in a practical setting.

\section{ACKNOWLEDGMENTS}

The first author, M. Jabbal, would like to acknowledge the financial support from the Engineering and Physical Sciences Research Council (EPSRC Grant No. AF566NEZ) for this work and the EPSRC Engineering Instrument Pool for the loan of the PIV system. The authors would also like to thank the former Goldstein Laboratory staff for technical support and Ms. Jue Zhou for the supporting numerical simulation.

${ }^{1}$ P. Lawrence, "Meeting the challenge of aviation emissions: An aircraft industry perspective," Tech. Analysis Strategic Management 21, 79 (2009).

${ }^{2}$ M. Amitay and A. Glezer, "Aerodynamic flow control using synthetic jet actuators," Control of Fluid Flow, Lecture Notes in Control and Information Sciences (Springer, New York, 2006).

${ }^{3}$ B. L. Smith, M. A. Trautman, and A. Glezer, "Controlled interactions of adjacent synthetic jets," AIAA Paper No. 1999-0669, 1999.

${ }^{4}$ M. Amitay, V. Kibens, D. Parekh, and A. Glezer, "Aerodynamic flow control over an unconventional airfoil using synthetic jet actuators," AIAA J. 39, 361 (2001).

${ }^{5}$ N. J. Wood, A. M. Sadri, and A. Crook, "Control of turbulent flow separation by synthetic jets," AIAA Paper No. 2000-4331, 2000.

${ }^{6}$ R. Mittal, P. Rampunggoon, and H. S. Udaykumar, "Interaction of a synthetic jet with a flat plate boundary layer," AIAA Paper No. 2001-2773, 2001.

${ }^{7}$ D. R. Smith, "Interaction of a synthetic jet with a crossflow boundary layer," AIAA J. 40, 2277 (2002).

${ }^{8}$ J. Cui, R. K. Agarwal, and A. W. Cary, "Numerical simulation of the interaction of a synthetic jet with a turbulent boundary layer," AIAA Paper No. 2003-3458, 2003.

${ }^{9}$ K. E. Wu and K. S. Breuer, "Dynamics of synthetic jet actuator arrays for flow control," AIAA Paper No. 2003-4257, 2003.

${ }^{10}$ J. M. Shuster, R. J. Pink, D. M. McEligot, and D. R. Smith, "The interaction of a circular synthetic jet with a cross-flow boundary layer," AIAA Paper No. 2005-4749, 2005.

${ }^{11}$ N. W. Schaeffler and L. N. Jenkins, "Isolated synthetic jet in crossflow: Experimental protocols for a validation dataset," AIAA J. 44, 2846 (2006).

${ }^{12}$ J. Dandois, E. Garnier, and P. Sagaut, "Unsteady simulation of a synthetic jet in crossflow," AIAA J. 44, 225 (2006).

${ }^{13} \mathrm{G}$. Hong, "Effectiveness of micro synthetic jet actuator enhanced by flow instability in controlling laminar separation caused by adverse pressure gradient," Sens. Actuators, A 132, 607 (2006).

${ }^{14}$ D. K. L. Wu and M. A. Leschziner, "Large-eddy simulations of circular synthetic jets in stagnant surroundings and turbulent cross-flow," IUTAM Symposium on Flow Control and MEMS (Springer, New York, 2008).

${ }^{15}$ S. Zhong, F. Millet, and N. J. Wood, "The behavior of circular synthetic jets in a laminar boundary layer," Aeronaut. J. 109, 461 (2005).

${ }^{16} \mathrm{M}$. Jabbal and S. Zhong, "The near wall effect of synthetic jets in a boundary layer," Int. J. Heat Fluid Flow 29, 119 (2008).

${ }^{17}$ S. Zhong, M. Jabbal, H. Tang, L. Garcillan, F. Guo, N. Wood, and C. Warsop, "Towards the design of synthetic-jet actuators for full-scale flight conditions. Part 1: The fluid mechanics of synthetic-jet actuators," Flow, Turbul. Combust. 78, 283 (2007).

${ }^{18} \mathrm{G}$. Godard and M. Stanislas, "Control of a decelerating boundary layer. Part 1: Optimization of passive vortex generators," Aerosp. Sci. Technol. 10, 181 (2006).

${ }^{19}$ W. R. Pauley and J. K. Eaton, "Experimental study of the development of longitudinal vortex pairs embedded in a turbulent boundary layer," AIAA J. 26, 816 (1988).

${ }^{20}$ K. A. Chang, T. J. Hsu, and P. L. F. Liu, "Generation and evolution in water waves propagating over a submerged obstacle. Part 1. Solitary waves," Coast. Eng. 44, 13 (2001).

${ }^{21}$ B. L. Smith and A. Glezer, "The formation and evolution of synthetic jets," Phys. Fluids 10, 2281 (1998).

${ }^{22} \mathrm{H}$. Tang and S. Zhong, "Incompressible flow model of synthetic jet actuators," AIAA J. 44, 908 (2006). 
${ }^{23}$ A. Glezer, "The formation of vortex rings," Phys. Fluids 31, 3532 (1988).

${ }^{24}$ J. Zhou and S. Zhong, "Numerical simulation of the interaction of a circular synthetic jet with a boundary layer," Comput. Fluids 38, 393 (2009).

${ }^{25} \mathrm{~S}$. Liddle, "The use of synthetic jet actuators to enhance deflected surface controls," Ph.D. thesis, University of Manchester, UK, 2007.

${ }^{26}$ R. S. Wakeland and R. M. Keolian, "Influence of velocity profile nonuni- formity on minor losses for flow exiting thermoacoustic heat exchangers," J. Acoust. Soc. Am. 112, 1249 (2002).

${ }^{27}$ R. Raju, Q. Gallas, R. Mittal, and L. Cattafesta, "Scaling of a pressure drop for oscillatory flow through a slot," Phys. Fluids 19, 078107 (2007).

${ }^{28}$ B. L. Smith and G. W. Swift, "Power dissipation and time-averaged pressure in oscillating flow through a sudden area change," J. Acoust. Soc. Am. 113, 2455 (2003). 